Artigo

\title{
Previsão Climática Sazonal para o Brasil Obtida Através de Modelos Climáticos Globais e Regional
}

\author{
Michelle Simões Reboita ${ }^{1}$ (id , Cássia Gabriele Dias ${ }^{1}$, Lívia Márcia Mosso Dutra ${ }^{2}$, \\ Rosmeri Porfírio da Rocha ${ }^{2}$, Marta Llopart ${ }^{3,4}$ \\ ${ }^{1}$ Instituto de Recursos Naturais, Universidade Federal de Itajubá, Itajubá, MG, Brasil. \\ ${ }^{2}$ Departamento de Ciências Atmosféricas, Universidade de São Paulo, São Paulo, SP, Brasil. \\ ${ }^{3}$ Departamento de Física, Faculdade de Ciências, \\ Universidade Estadual Paulista Júlio de Mesquita Filho, Bauru, SP, Brasil. \\ ${ }^{4}$ Centro de Meteorologia de Bauru, Bauru, SP, Brasil.
}

Recebido em 14 de Junho de 2017 - Aceito em 20 de Setembro de 2017.

\begin{abstract}
Resumo
Este estudo avalia a destreza de dois modelos climáticos globais (CPTEC e CFSv2) e de um modelo climático regional (RegCM4) em prever o clima sazonal em diferentes regiões do Brasil. O RegCM4 foi dirigido tanto com as saídas do sistema CFSv2 quanto do modelo do CPTEC. Também foram utilizadas duas versões do RegCM: a 4.3 e a 4.5. O RegCM4.3 foi dirigido por seis membros do CFSv2, enquanto o RegCM4.5 foi dirigido por um membro do modelo global do CPTEC. Todas as previsões iniciaram cerca de dois meses antes do trimestre a ser previsto e ao todo foram realizadas 94 simulações com o modelo regional. De forma geral, o RegCM4 adiciona valor às previsões dos modelos climáticos globais, principalmente, quando é aninhado às saídas do modelo do CPTEC. Quando o RegCM4.5 é dirigido por esse modelo global e é utilizada a parametrização de convecção cumulus de Emanuel há uma boa performance do modelo regional na previsão da precipitação e temperatura do ar em quase todo o Brasil.

Palavras-chave: modelo climático regional, modelo climático global, ensemble, Brasil.
\end{abstract}

\section{Seasonal Climate Forecast to Brazil Obtained Through Global and Regional Climate Models}

\begin{abstract}
This study evaluates the ability of two global climate models (CPTEC and CFSv2) and a regional climate model (RegCM4) in predicting the seasonal climate in different regions of Brazil. RegCM4 was drove with the outputs of two global climate models: CFSv2 system and CPTEC. Two versions of RegCM were employed in the study: 4.3 and 4.5 . RegCM4.3 was ran with six members of CFSv2 system, while RegCM4.5 was ran with a member of the CPTEC model. All forecasts started about two months before the season to be predicted and in all 94 simulations with the regional climate model were carried out. RegCM4 adds value to the predictions of the global climate models, mainly when it is nested to the CPTEC outputs. When RegCM4.5 is drove by CPTEC outputs and Emanuel cumulus convection parameterization is used, there is a good performance of the regional climate model in predicting the precipitation and air temperature in almost all Brazil.
\end{abstract}

Keywords: regional climate model, global climate model, ensemble, Brazil.

\section{Introdução}

Diferente da previsão de tempo, em que se deseja saber como serão as condições atmosféricas nos dias seguintes, a previsão climática aponta se uma dada estação do ano será, por exemplo, mais seca ou chuvosa do que a média climatológica, mais quente ou mais fria etc., bem como a intensidade das anomalias em relação à média (Ynoue et al., 2017).

A previsão climática sazonal pode ser realizada com modelos de circulação geral da atmosfera ou com modelos

Autor de correspondência: Michelle S. Reboita, reboita@gmail.com. 
de área limitada, também chamados de modelos climáticos regionais (MCR). Os MCR, em geral, produzem prognósticos mais acurados do que os modelos globais, já que possuem melhor resolução horizontal e parametrizações físicas mais adequadas para resolver os processos atmosféricos de mesoescala (Giorgi e Mearns, 1999; Misra et al., 2003; Cuadra e da Rocha, 2006). Portanto, essas características dos MCR podem contribuir para previsões mais acuradas em regiões de topografia complexa como, por exemplo, no sudeste do Brasil.

A previsão climática é muito mais difícil de ser realizada do que a de tempo, pois necessita de condição inicial acurada da atmosfera (de fronteiras também no caso dos MCR), bem como da especificação da vegetação e inicialização da temperatura da superfície do mar (TSM) e umidade do solo, por afetarem diretamente as trocas que ocorrem entre a superfície e a atmosfera (Llopart et al., 2017). Destaca-se aqui que a sazonalidade do armazenamento de água no solo introduz um "efeito de memória" da ordem de um a dois meses (Koster e Suarez, 2001; Seneviratne et al., 2006; Dirmeyer et al., 2009; Orlowsky e Seneviratne 2010; Koster et al., 2011), de forma que a inicialização e a previsão errônea dessa variável em um modelo de clima pode causar inconsistências na simulação dos fluxos de superfície, fazendo com que o modelo não seja capaz de prever adequadamente a precipitação e a temperatura do ar em escalas de tempo mais curtas (van den Hurk et al., 2012). Este fato justifica a necessidade de uma inicialização das características da superfície continental de forma mais realística possível nos modelos climáticos (Doblas-Reyes et al., 2013a,b). O período para ajuste do ciclo hidrológico nos modelos climáticos (mínimo de um mês de antecedência do período a ser previsto) é chamado de spin-up (Giorgi e Mearns, 1999). Também é importante ressaltar que a previsão climática sazonal envolve períodos superiores a 15 dias o que também está suscetível ao efeito do caos (Lorenz, 1965).

Os MCR são dirigidos por dados de reanálise ou de simulação de modelos globais. Dentre os modelos climáticos globais usados na previsão sazonal, podem-se citar o sistema Climate Forecast System version 2 - CFSv2 (Saha et al., 2014) do National Centers for Environmental Prediction (Estados Unidos) e o modelo global do Centro de Previsão de Tempo e Estudos Climáticos - CPTEC (Cavalcanti et al., 2002; Marengo et al., 2003; Panetta et al., 2006). Esses dois modelos globais são utilizados no presente estudo.

As previsões de precipitação do sistema CFSv2 foram analisadas por Reboita et al. (2011), no período entre 1999 e 2010 sobre a América do Sul (AS), através de comparações com as análises do Global Precipitation Climatology Project (GPCP) e do Climate Prediction Center Merged Analysis of Precipitation (CMAP). Segundo os autores, o CFSv2 simula o padrão espacial da precipitação anual e sazonal semelhante aos das análises, mas, em geral, subestima a precipitação sobre o continente em cerca de 1-2 $\mathrm{mm} /$ dia e superestima na Zona de Convergência Intertropical (ZCIT). Esses autores também concluíram que o CFSv2 é hábil em reproduzir a variação sazonal da precipitação na AS e, portanto, suas variáveis simuladas (temperatura, vento etc.) podem ser utilizadas como condições inicial e de fronteira em MCR. Já Silva et al. (2014) analisaram hindcasts do CFSv2 no período de dez-jan-fev (DJF) e jun-jul-ago (JJA) de 1983 a 2010 sobre a AS. Entre os resultados foi verificado que o CFSv2 representa melhor a precipitação sobre o continente do que sobre o oceano.

De acordo com Cavalcanti et al. (2002), o modelo global do CPTEC reproduz bem o ciclo sazonal das principais variáveis atmosféricas, mas com alguns erros sistemáticos. O modelo superestima (subestima) a precipitação na porção sul (norte) das zonas de convergência no Hemisfério Sul. Entretanto, representa adequadamente as altas subtropicais, o jato de altos níveis e os storm tracks. Mais recentemente, Coelho et al. (2012) verificaram a performance do modelo do CPTEC em prever o período seco da monção Sul Americana no trimestre de julho a setembro dos anos de 1998, 2005 e 2010, quando ocorreram eventos de seca na Amazônia. O modelo previu as condições secas nesses anos, particularmente no oeste da Amazônia. Os autores ainda destacaram que o resultado do modelo foi importante para alertar previamente os tomadores de decisão sobre a seca na Amazônia.

No Brasil, o Centro de Previsão de Tempo e Estudos Climáticos (CPTEC) utiliza dois modelos numéricos para fornecer previsões climáticas: o modelo de circulação geral da atmosfera, CPTEC (Cavalcanti et al., 2002), e o modelo regional Eta (Chou et al., 2005). Chou et al. (2000, 2005) mostraram que o modelo Eta, dirigido pelas simulações do modelo de circulação geral do CPTEC, reproduz melhor a precipitação do que este modelo global, ou seja, há agregação de valor na simulação com o modelo regional. Embora, o modelo Eta melhora as previsões climáticas comparado ao modelo global do CPTEC, ainda superestima a precipitação, principalmente, entre os meses de agosto a novembro e as áreas com maiores erros nas previsões estendem-se ao longo das costas norte e nordeste do Brasil e sobre os Andes. Na região sudeste do Brasil, as maiores superestimativas ocorrem no sul de Minas Gerais (Chou et al., 2005).

A previsão climática sazonal que é divulgada à sociedade brasileira provém de uma reunião entre o Grupo de Trabalho em Previsão Climática Sazonal do Ministério da Ciência, Tecnologia, Inovações e Comunicações (GTPCS/MCTIC) com meteorologistas do CPTEC-INPE, Instituto Nacional de Meteorologia (INMET), Fundação Cearense de Meteorologia e Recursos Hídricos e Centros Estaduais de Meteorologia. Nessa reunião, os meteorologistas analisam as previsões numéricas geradas pelos modelos do CPTEC e de outros centros internacionais de meteorologia e através da junção de conhecimentos sobre o 
estado atual do clima e específicos inerentes à meteorologia geram uma previsão por consenso que é divulgada, por exemplo, no setor Clima do sítio do CPTEC. Nessa mesma linha, o Grupo de Estudos Climáticos da Universidade de São Paulo (GrEC-USP) juntamente com pesquisadores de outras universidades do Brasil, como da Universidade Federal de Itajubá (UNIFEI), realizam trimestralmente previsões climáticas para o Brasil com base em produtos numéricos gerados por diferentes centros de meteorologia. Entretanto, um dos objetivos desse grupo é um estudo da qualidade das previsões climáticas sazonais do Regional Climate Model (RegCM; Giorgi et al., 2012) a fim de ser utilizado de forma operacional. Com ênfase nesse objetivo, Machado e da Rocha (2011) verificaram o desempenho do RegCM3 aninhado ao modelo global do CPTEC sobre o Brasil, entre maio de 2005 e julho de 2007. De acordo com os autores, as previsões de chuva e temperatura do RegCM3 são mais próximas das observações do que as do CPTEC. O RegCM3 também foi capaz de corrigir alguns erros sistemáticos do modelo global, portanto, adicionando valor às previsões regionais, o que indicou um possível potencial para ser empregado na previsão sazonal.

Reboita (2013) avaliou a performance de diferentes esquemas de parametrização de convecção cumulus (Grell-FC, Grell-AS, Kuo, Emanuel-MIT, Mixed1 e Mixed2) em prever as condições climáticas sazonais no Brasil quando o RegCM4 era aninhado às previsões do modelo global do CPTEC e do sistema CFSv2. Foram realizados prognósticos para três trimestres do ano de 2012 (MAM, AMJ e SON) o que resultou em 48 simulações com o RegCM4. Destas, em 36 o RegCM4 foi dirigido pelo modelo global do CPTEC e em 12 pelo modelo CFSv2. A comparação das simulações dirigidas pelo modelo do CPTEC com as dirigidas pelo sistema CFSv2 no trimestre SON/2012 mostrou que as condições de fronteira exercem grande influência nos resultados das simulações regionais. Por exemplo, as fronteiras provenientes do modelo global do CPTEC favoreceram a ocorrência de um intenso viés negativo de precipitação entre o oeste da Amazônia e o centro-norte de Minas Gerais nas previsões com todos os seis esquemas de parametrização de convecção cumulus utilizados. Além disso, propiciaram a ocorrência de viés positivo de temperatura em grande parte do Brasil. Por outro lado, as fronteiras provenientes do sistema CFSv2 favoreceram um viés positivo de precipitação entre o oeste da Amazônia e o centro-norte de Minas Gerais e um viés negativo de temperatura do ar em quase todo o domínio. Em geral, o esquema Mixed1 (Grell sobre o continente e Emanuel sobre o oceano) produz prognósticos ligeiramente mais similares às observações do que os demais esquemas de convecção cumulus para as diferentes regiões do Brasil. Reboita et al. (2014) testaram diferentes combinações de parametrizações físicas do RegCM4 sobre a AS e também destacaram boa performance do esquema de convecção Mixed1 com o esquema de superfície BATS. Outros estu- dos, como da Rocha (2005), também indicam boa performance do RegCM3 em simular o clima da AS com o esquema de parametrização cumulus de Grell com fechamento de Fritsch-Chapell (FC).

Gonçalves (2015) dirigiu o RegCM4 com o CFSv2 para avaliar a previsão climática sazonal de precipitação no Estado do Rio Grande do Sul, no período de agosto de 2013 a agosto de 2014. Foram realizadas 34 simulações de 5 meses de duração para cada uma das 9 opções de parametrizações de convecção cumulus utilizadas no estudo. Os dois primeiros meses de cada previsão foram considerados como período de spin-up. O RegCM4, em geral, subestimou a precipitação nas regiões centro-leste, sul-sudoeste e norte-nordeste do Rio Grande do Sul com a maioria das parametrizações. Já nas regiões oeste e noroeste ocorreu superestimativa de precipitação. Apesar do RegCM4 não representar adequadamente a precipitação, mesmo assim, teve habilidade em reduzir as superestimativas de precipitação ocorridas com o CFSv2. Estudos como o de Reboita et al. (2014), Llopart et al. (2014) e de Jesus et al. (2016) também confirmam o viés seco de diferentes versões do RegCM sobre o sul do Brasil e bacia do Prata, que é uma deficiência do modelo regional.

O último relatório do Painel Intergovernamental sobre Mudanças Climáticas (IPCC, 2013) enfatiza a necessidade de se prever o clima em curto prazo a fim de se ter informações consistentes para tomadores de decisão (governantes). No Brasil, por exemplo, a agricultura, geração de energia hidroelétrica e turismo são algumas das atividades econômicas diretamente influenciadas pelo clima. Assim, previsões climáticas de boa qualidade se tornam de grande importância para o sucesso dessas atividades.

Considerando que prognósticos mais precisos do clima podem auxiliar no planejamento de atividades de diferentes setores da sociedade e tendo em vista um complemento aos esforços da comunidade científica na realização de previsões climáticas sazonais para o Brasil, esse estudo objetiva aprofundar o conhecimento da qualidade das previsões climáticas sazonais dos modelos climáticos globais CFSv2 e CPTEC e do RegCM4 sobre o Brasil.

\section{Metodologia}

\subsection{Modelos globais}

O sistema CFSv2 do NCEP é um modelo acoplado que representa a interação dos oceanos, superfície e atmosfera (Saha et al., 2014). A componente atmosférica do CFSv2 é o modelo Global Forecast System (GFS; Moorthi et al., 2001) e a oceânica é o modelo Modular Ocean Model version 3 (MOM3, Pacanowski e Griffes, 1998). As previsões da componente atmosférica do CFSv2 são disponibilizadas com resolução horizontal de $0,9^{\circ} \times 0,9^{\circ}$ de latitude e longitude e com 37 níveis verticais em coordenada de pressão. 
O modelo global do CPTEC é atmosférico e espectral sendo executado com resolução horizontal aproximada de $1,875^{\circ} \times 1,875^{\circ}$ de latitude por longitude e com 28 níveis verticais. O esquema de parametrização de superfície utilizado é o SSiB (Xue, 1991) e o de convecção cumulus de Kuo. Enquanto as previsões do CFSv2 são atualizadas diariamente na internet podendo ser obtidas por qualquer pesquisador, as do CPTEC devem ser solicitadas.

\subsection{Modelo regional}

O RegCM foi desenvolvido em 1989 pelo National Center for Atmospheric Research (NCAR) e é mantido atualmente pelo Earth System Physics (ESP) do Abdus Salam International Centre for Theoretical Physics (ICTP). Foi o primeiro modelo de área limitada aplicado a estudos climáticos (Giorgi e Mearns, 1999; Giorgi e Anyah, 2012). O RegCM4 é um modelo climático de área limitada, compressível e em coordenada vertical sigmapressão. Atualmente o modelo possui versão hidrostática e não-hidrostática (Elguindi et al., 2014). No presente estudo foi empregada a versão hidrostática. Uma descrição detalhada da dinâmica e dos processos físicos do RegCM4 é fornecida em Giorgi et al. (2012). Já a descrição detalhada das simulações é apresentada no item 2.3.

No presente estudo foram utilizadas duas versões do RegCM: o RegCM4.3 e o RegCM4.5. Essa última versão foi lançada em 2016, quando as simulações do RegCM4.3 aninhado ao CFSv2 já haviam sido realizadas. Portanto, devido ao tempo de processamento e espaço necessário para armazenar os dados, decidiu-se utilizar a versão 4.5 apenas para as simulações aninhadas às saídas do modelo do CPTEC que ainda não haviam sido realizadas.

\subsection{Características das simulações climáticas}

As simulações climáticas foram realizadas para um domínio que inclui a AS e oceanos adjacentes (Fig. 1) e inicializadas cerca de dois meses antes do período a ser previsto. Esse início prévio permite que o modelo ultrapasse o período de spin-up, que para a atmosfera é de poucos dias, mas para os processos no solo é mais lento (Giorgi e Mearns, 1999). O modelo foi integrado com $30 \mathrm{~km}$ de resolução horizontal e 18 níveis verticais em coordenada sigma-pressão.

O RegCM4.3 foi dirigido por previsões do ano de 2013 e início de 2014 de seis membros do sistema CFSv2 (Saha et al., 2014) com início em datas diferentes (Tabela 1). $\mathrm{O}$ ano de 2013 foi escolhido por não apresentar

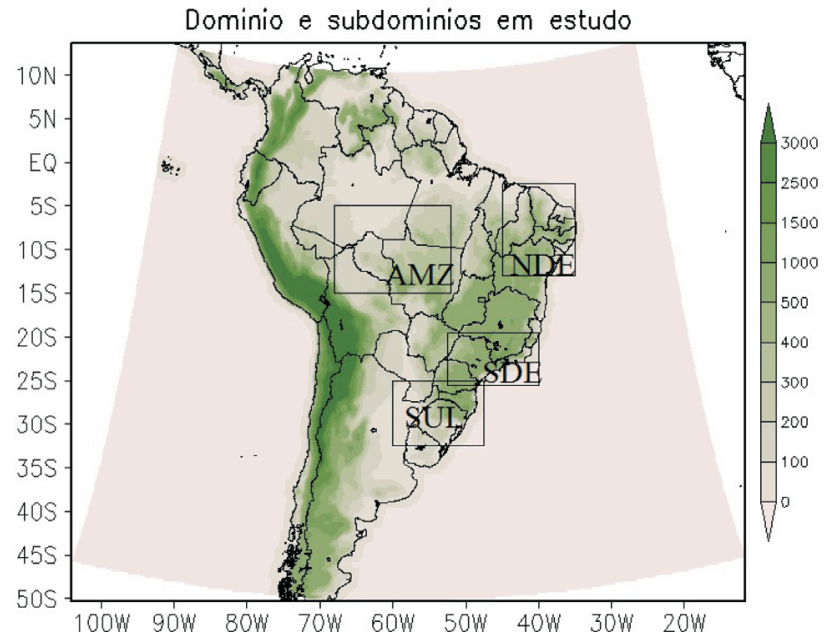

Figura 1 - Domínio e topografia (metros) das simulações com o RegCM4. Também são apresentados os subdomínios de estudo: Amazônia (AMZ), Nordeste (NDE), Sudeste (SDE) e Sul (SUL).

grandes anomalias climáticas (Blunden e Arndt, 2014), o que é oposto ao ocorrido nos meses de janeiro e fevereiro de 2014, quando uma grande seca atingiu o sudeste do Brasil (Reboita et al., 2015a, Coelho et al., 2016). Como foram realizadas previsões para 12 trimestres e iniciando em 6 datas distintas, ao todo obtiveram-se 72 simulações com o RegCM4 aninhado ao CFSv2. Nestas previsões foram utilizadas as parametrizações físicas: BATS (Dickinson et al., 1993) para descrever a interação superfície-atmosfera e Grell (1993) com fechamento de Fritsch-Chapell (GrellFC) para a convecção cumulus. As previsões do RegCM4.3 serão chamadas de RegCFSv2.

O RegCM4.5 também foi dirigido por previsões do ano de 2013 e início de 2014 (Tabela 1), mas por apenas um membro do modelo global do CPTEC (Cavalcanti et al., 2002), já que num teste preliminar em que o RegCM4.5 foi executado com diferentes datas de início de condições iniciais, não apresentou uma grande dispersão entre os membros. Entretanto, foram realizados dois conjuntos de simulações: um empregando a parametrização cumulus de Emanuel (Emanuel e Zivkovic-Rothman, 1999) e outro em que se utilizou uma nova parametrização, o esquema de Kain-Fritsch (Kain, 2004). Assim, essas simulações foram denominadas de RegCPTECEman e RegCPTECKF, respectivamente. Como as previsões com o RegCM4.5 aninhado às saídas do modelo CPTEC incluíram somente um membro, foram realizadas 11 previsões utilizando a para-

Tabela 1 - Detalhamento das datas de início das previsões com o RegCM4.

\begin{tabular}{lclcl}
\hline Versão do RegCM & Modelo Global & Datas de início das simulações & Horário de Início (UTC) & Trimestres simulados \\
\hline RegCM4.3 & CFSv2 & $\begin{array}{l}1,5,10,15,20 \text { e } 25 \text { de dois meses } \\
\text { prévios ao trimestre simulado }\end{array}$ & 00 & $\begin{array}{l}\text { mar/abr } / \mathrm{mai} 2013 \text { até fev } / \mathrm{mar} / \mathrm{abr} \\
2014\end{array}$ \\
RegCM4.5 & CPTEC & $\begin{array}{l}15 \text { de dois meses prévios ao } \\
\text { trimestre simulado }\end{array}$ & 12 & $\begin{array}{l}\text { mar/abr } / \mathrm{mai} 2013 \text { até fev } / \mathrm{mar} / \mathrm{abr} \\
2014\end{array}$ \\
\hline
\end{tabular}


metrização de cumulus Kain-Fritsch e 11 com a de Emanuel, pois não se obtiveram as condições iniciais e de fronteira do mês de outubro de 2013.

Para as simulações com o RegCM4.3 e 4.5, foram utilizados sobre o continente dados de topografia e uso do solo do United States Geological Survey (USGS) e do Global Land Cover Characterization (GLCC), respectivamente, com 10' de resolução horizontal descritos em Loveland et al. (2000). A condição inicial da umidade do solo no modelo seguiu a especificação do tipo de vegetação (Giorgi e Bates, 1989). Com relação à temperatura da superfície do mar (TSM), na previsão do modelo regional dirigido pelas saídas do sistema CFSv2 esta é prevista, enquanto a dirigida pelo modelo do CPTEC é persistida. Coelho et al. (2012) explica como é utilizada a TSM persistida no modelo global do CPTEC com o seguinte exemplo: para uma previsão iniciada em junho de 2010 e válida para julho a setembro de 2010, a anomalia de TSM do mês de maio de 2010 é adicionada a média climatológica da TSM em cada mês do período de junho a setembro.

\subsection{Previsão por conjunto (ensemble)}

Considerando que um modelo é a representação aproximada de um sistema e, no caso, que os modelos climáticos são modelos numéricos usados para calcular a quantia de massa, momentum e energia com base em leis físicas de conservação (Gettelman e Rood, 2016), logo existem incertezas associadas aos resultados produzidos pelos modelos. As incertezas podem ser decorrentes do próprio modelo (parametrizações físicas, arredondamentos etc.), do cenário utilizado e das condições iniciais. Uma maneira de quantificar as incertezas é através da realização de várias simulações (ensembles) de um determinado período e computar a média dessas simulações. As simulações podem iniciar em datas diferentes (ensemble temporal), na mesma data, mas alterando as parametrizações físicas do modelo (ensemble físico), mesclando ambos os tipos (ensemble temporal e físico), executando simulações com diferentes modelos etc. A técnica de ensemble em português é chamada de previsão numérica por conjunto. Entretanto, por brevidade, será utilizada no estudo a denominação ensemble.

No presente estudo são realizados ensembles temporais das previsões dos modelos globais (CFSv2 e CPTEC) e das previsões do RegCM4.3 dirigido pelo CFSv2 (Tabela 1). O ensemble das previsões do sistema CFSv2 incluiu 6 membros, sendo as datas de início das simulações os dias $1,5,10,15,20$ e 25 de cerca de dois meses prévios ao trimestre a ser previsto. Já o ensemble das previsões do modelo global do CPTEC foi realizado com 5 membros que tiveram início nos dias 13, 14, 15, 16 e 17, também de cerca de dois meses prévios ao trimestre a ser previsto. No caso das previsões do RegCM4.3 dirigido pelo CFSv2, as datas de início das simulações são as mesmas do sistema CFSv2. Recorda-se aqui que o RegCM4.5 só foi aninhado a um membro do modelo do CPTEC (dia 15 foi usado para início das previsões com o modelo regional) e, por isso, não há ensemble.

\subsection{Validação das previsões sazonais}

Foram avaliadas as médias trimestrais das variáveis atmosféricas temperatura do ar a $2 \mathrm{~m}$ de altura e precipitação, tanto em relação à distribuição espacial quanto em valores médios em 4 regiões sobre o Brasil (Fig. 1; Tabela 2): Amazônia (AMZ), Nordeste (NDE), Sudeste (SDE) e Sul do Brasil (SUL). Essa avaliação foi realizada tanto para as previsões dos modelos globais quanto para as simulações com o RegCM4.

As médias da precipitação simulada foram comparadas com os dados do Climate Prediction Center (CPC; Chen et al., 2008) e com as observações do Tropical Rainfall Measuring Mission (TRMM; Huffman et al., 2007). Os dados do CPC cobrem apenas o continente e possuem resolução horizontal de $0,5^{\circ} \times 0,5^{\circ}$ de latitude e longitude. Além disso, o CPC é o único conjunto de dados atualizado diariamente. Já o TRMM possui resolução horizontal de $0,25^{\circ} \times 0,25^{\circ}$. Para a validação da temperatura do ar utilizou-se a reanálise ERA-Interim (Dee et al., 2011) com resolução horizontal de $0,75^{\circ} \times 0,75^{\circ}$ e a reanálise CFSR com resolução horizontal de $0,3125^{\circ} \times 0,3125^{\circ}$. Até a data da obtenção da reanálise CFSR, só havia dados disponíveis até 2013. Utilizaram-se dois conjuntos de dados para a validação de cada previsão, pois, em geral, os dados de diferentes fontes também apresentam algumas diferenças entre eles (da Rocha et al., 2014).

As médias da distribuição espacial das variáveis atmosféricas previstas foram interpoladas com o método bi-linear (Press et al., 2007) para a mesma resolução das observações a que são comparadas, a fim de se calcular o viés: simulação menos observação. $\mathrm{O}$ viés também foi computado para os subdomínios da Tabela 2, bem como o índice de concordância de Willmott (Willmott, 1981), que mede o grau de concordância entre os valores previstos e observados. Quando o resultado é 1 indica concordância perfeita, enquanto que 0 indica que não há concordância entre previsão e observação. $O$ índice de concordância de Willmott (d) é calculado pela seguinte equação:

$$
d=1-\frac{\sum_{i=1}^{n}\left(P_{i}-O_{i}\right)^{2}}{\sum_{i=1}^{n}\left(\left|P_{i}^{\prime}\right|+\left|O_{i}^{\prime}\right|\right)^{2}}
$$

Tabela 2 - Localização dos subdomídios de estudo.

\begin{tabular}{lcc}
\hline Subdomínio & Latitude & Longitude \\
\hline AMZ & $15^{\circ} \mathrm{S}$ a $5^{\circ} \mathrm{S}$ & $68^{\circ} \mathrm{W}$ a $52^{\circ} \mathrm{W}$ \\
$\mathrm{NEB}$ & $13^{\circ} \mathrm{S}$ a $2,5^{\circ} \mathrm{S}$ & $45^{\circ} \mathrm{W}$ a $35^{\circ} \mathrm{W}$ \\
$\mathrm{SDE}$ & $25,5^{\circ} \mathrm{S}$ a $19,5^{\circ} \mathrm{S}$ & $52,5^{\circ} \mathrm{W}$ a $40^{\circ} \mathrm{W}$ \\
$\mathrm{SUL}$ & $32,5^{\circ} \mathrm{S}$ a $25^{\circ} \mathrm{S}$ & $60^{\circ} \mathrm{W}$ a $47,5^{\circ} \mathrm{W}$ \\
\hline
\end{tabular}


onde $P_{i}=$ valor previsto; $O_{i}=$ valor observado, $P_{i}=P_{i}-\bar{O}$, $O_{i}=O_{i}-\bar{O}$ e $\bar{O}=$ média dos valores observados.

\subsection{O verão de 2014}

A precipitação nos meses de janeiro e fevereiro de 2014 foi abaixo da normal climatológica no sudeste do Brasil (Reboita et al., 2015a; Coelho et al., 2016). Marengo et al. (2015) ressaltam que foram observados déficits de precipitação entre janeiro e março sobre o sudeste do Brasil de 150 a $200 \mathrm{~mm} / \mathrm{mês}$ abaixo da normal. Esses valores concordam com Coelho et al. (2016) que afirmam que em algumas áreas dentro desta região o déficit foi superior a $300 \mathrm{~mm}$ durante este período. Reboita et al. (2015a) compararam a precipitação de janeiro de 2014 com a média do período de 1979 a 2015 deste mesmo mês e notaram que há vários janeiros nos quais a anomalia de precipitação foi negativa no sudeste do Brasil, entretanto, os anos mais secos foram 2014 e 2015. O déficit de precipitação no verão de 2014 gerou uma grande crise hídrica em relação ao abastecimento da população como relatam Marengo et al. (2015).

Diante desse contexto, foi verificado se o sistema CFSv2 e o RegCM4.3 conseguiram prever a seca de 2014 no sudeste do Brasil e realizada uma análise da circulação atmosférica desses modelos.

\subsection{Períodos secos e úmidos}

Também se investigou a performance das simulações em prever períodos secos e úmidos consecutivos, dentro de um dado trimestre, através de comparação com as observações do CPC e TRMM. Em cada subdomínio (Fig. 1), foi calculado o percentil de $10 \%$ e $90 \%$ por trimestre, a partir dos dados diários, e os valores obtidos foram utilizados como limiares para a definição dos períodos secos e úmidos, respectivamente (Reboita et al., 2013). É importante ressaltar que dias sem precipitação não entram na análise. Foi estabelecido no mínimo 3 dias com valor menor (maior) do que o limiar de 10\% (90\%) para a ocorrência de períodos secos (úmidos). Nesta análise, está sendo verificado se as previsões acertaram o número de períodos secos e úmidos, e não as datas de ocorrência.

\section{Resultados}

\subsection{Previsões sazonais para a precipitação e temperatura do ar a 2 m}

Embora tenham sido analisados todos os trimestres indicados na Tabela 1, por brevidade, as Figs. 2 e 3 apresentam somente os trimestres que correspondem exatamente às estações do ano (MAM, JJA e SON, porém, JFM, para o verão). $\mathrm{O}$ viés da precipitação ( $\mathrm{mm} /$ dia) entre a previsão do CPTEC, RegCPTECKF e RegCPTECEman em relação à análise do CPC e do TRMM é mostrado na Fig. 2, enquanto o viés da previsão CFSv2 e RegCFSv2, na Fig. 3. A Fig. 2 está organizada da seguinte maneira: o viés da previsão entre o CPTEC e CPC (CPTEC-CPC) é mostrado na primeira linha, do RegCPTECKF-CPC na segunda, do RegCPTECEman-CPC na terceira, do CPTEC-TRMM na quarta, do RegCPTECKF-TRMM na quinta e do RegCPTECEman-TRMM na sexta linha. Já na Fig. 3, o viés da previsão CFSv2-CPC é mostrado na primeira linha, do RegCFSv2-CPC na segunda, do CFSv2-TRMM na terceira e do RegCFSv2-TRMM na quarta linha. Em ambas as figuras as colunas indicam as estações do ano. Como o CPTEC, RegCPTECKF e RegCPTECEman não possuem a rodada de outubro (a previsão do modelo global ficou corrompida ao transferir os dados de uma máquina para outra), que é referente à previsão de DJF (verão), foi utilizado o trimestre JFM em todas as figuras, para permitir uma análise comparativa entre as previsões. Em todos os trimestres, os vieses das previsões da precipitação são mais acentuados em relação ao TRMM (Figs. 2 e 3 ).

No trimestre MAM (Figs. 2 e 3 a, e, i, m, q, u), a melhor previsão sobre o Brasil foi a do CFSv2 seguida do RegCFSv2 (Fig. 3). As previsões RegCPTECKF e RegCPTECEman (Fig. 2), no trimestre de MAM, aumentam os vieses secos, comparadas ao modelo do CPTEC, sobre o sul do Brasil e reduzem o viés úmido no nordeste do país. Em MAM, o RegCFSv2 mostra melhor resultado em relação ao CFSv2 somente no setor oeste da $\mathrm{AS}$, onde reduz o viés úmido (Fig. 3). Já no sul do Brasil, o RegCFSv2 muda o sinal do viés apresentado no CFSv2, de positivo para negativo, e aumenta o positivo no nordeste do Brasil.

No trimestre JJA (Figs. 2 e 3 b, f, j, n, r, v), que corresponde à estação seca no sudeste e centro-oeste do Brasil (Reboita et al., 2010), em geral, os modelos preveem bem esse sinal, exceto pelas previsões CPTEC e RegCPTECEman (Fig. 2) apresentarem um viés úmido em Minas Gerais e seco em Mato Grosso do Sul. Um resultado interessante é que as previsões RegCPTECKF e RegCPTECEman reduzem o viés úmido do modelo global do CPTEC sobre o nordeste do Brasil, o que adiciona valor ao modelo regional. Já o RegCFSv2 (Fig. 3) transforma o viés úmido do CFSv2 sobre o sul do Brasil em viés seco e o viés seco do norte do Brasil em úmido. Nesse trimestre, a melhor previsão foi a do RegCPTECKF seguida do RegCPTECEman e RegCFSv2.

Em SON inicia a estação chuvosa em grande parte do Brasil (Reboita et al., 2010). Nessa estação (Figs. 2 e 3 c, g, k, o, s, w), os modelos globais e a previsão RegCPTECEman são os que apresentaram as melhores performances na previsão da precipitação. No RegCPTECKF (Fig. 2) predomina viés seco em quase todo o continente, exceto no norte da AS. Já no modelo do CPTEC e no RegCPTECEman, os vieses são menores sobre a região centro-oeste e norte do país comparados às demais previsões. Por outro lado, o RegCFSv2 muda o sinal do viés mostrado no CFSv2, isto é, simula condições 

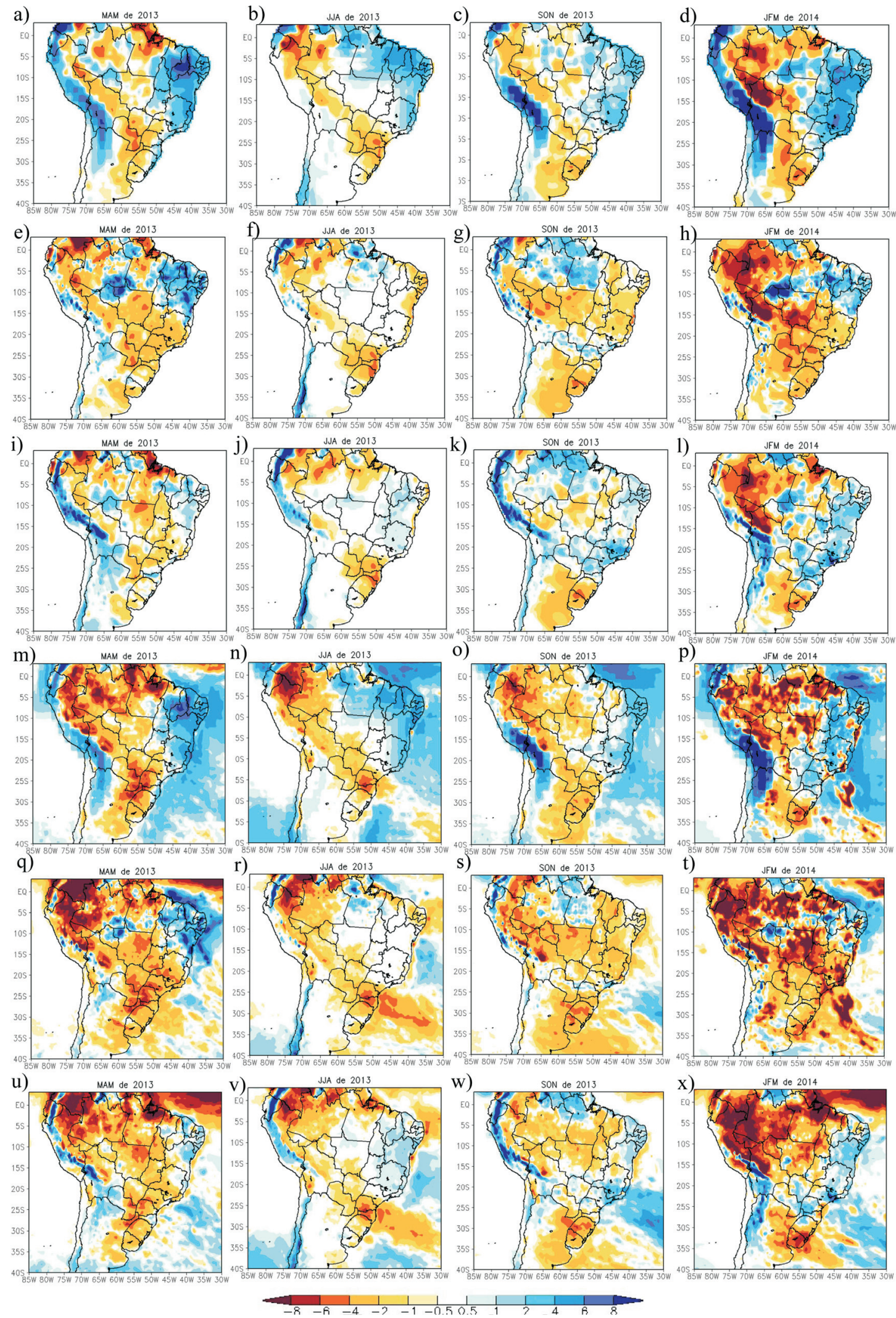

Figura 2 - Viés da precipitação (mm/dia): CPTEC menos CPC (primeira linha): a) MAM de 2013, b) JJA de 2013, c) SON de 2013 e d) JFM de 2014. RegCPTECKF menos CPC (segunda linha): e) MAM de 2013, f) JJA de 2013, g) SON de 2013 e h) JFM de 2014. RegCPTECEman menos CPC (terceira linha): i) MAM de 2013, j) JJA de 2013 e k) SON de 2013 e l) JFM de 2014. CPTEC menos TRMM (quarta linha): m) MAM de 2013, n) JJA de 2013, o) SON de 2013 e p) JFM de 2014. RegCPTECKF menos TRMM (quinta linha): q) MAM de 2013, r) JJA de 2013, s) SON de 2013 e t) JFM de 2014. RegCPTECEman menos TRMM (sexta linha): u) MAM de 2013, v) JJA de 2013 e w) SON de 2013 e x) JFM de 2014. 

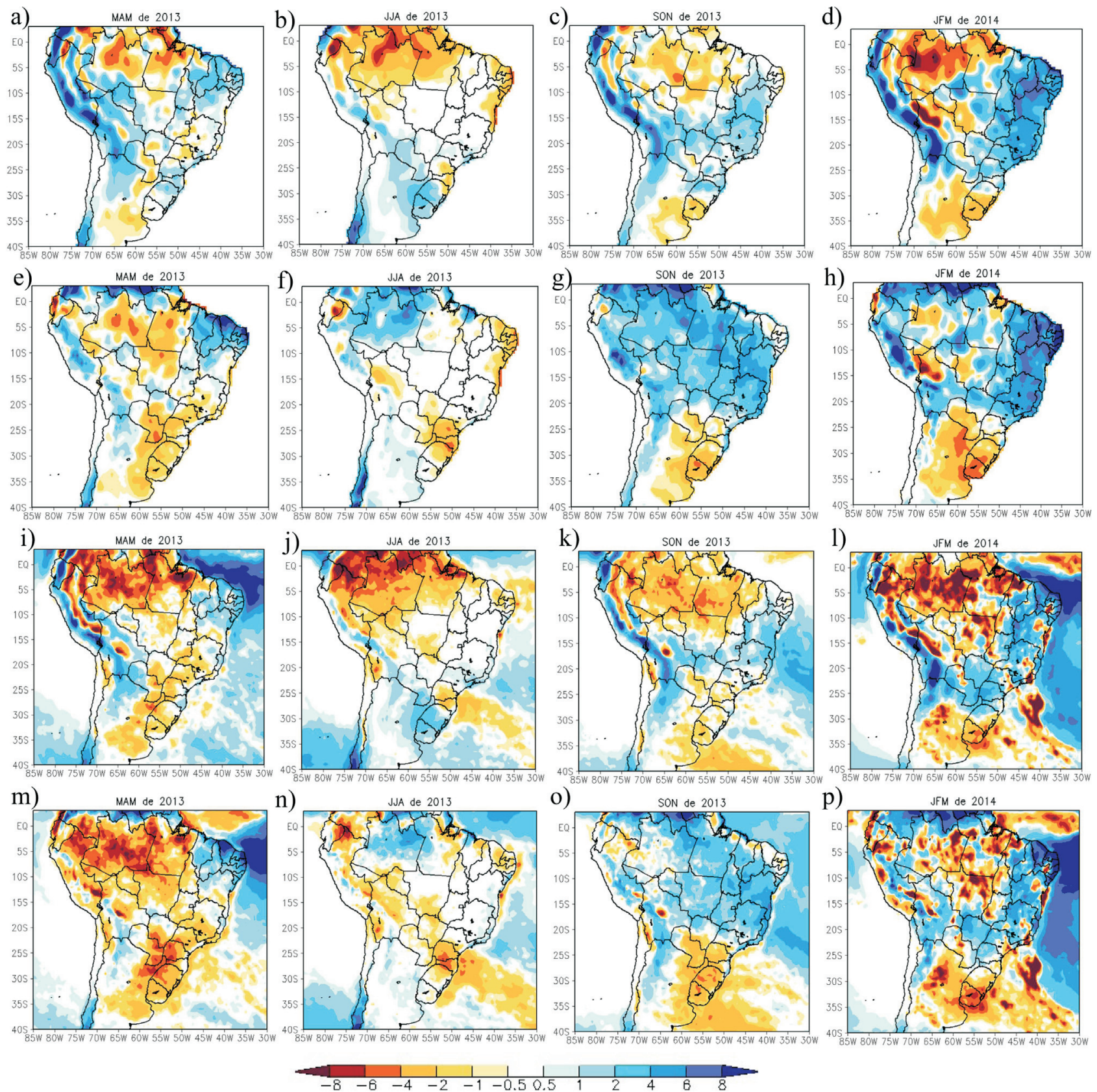

Figura 3 - Viés da precipitação (mm/dia): CFSv2 menos CPC (primeira linha): a) MAM de 2013, b) JJA de 2013, c) SON de 2013 e d) DJF de $2013 / 2014$. RegCFSv2 menos CPC (segunda linha): e) MAM de 2013, f) JJA de 2013, g) SON de 2013 e h) DJF de 2013/2014. CFSv2 menos TRMM (terceira linha): i) MAM de 2013,j) JJA de 2013 e k) SON de 2013 e l) DJF de 2013/2014. RegCFSv2 menos TRMM (quarta linha): m) MAM de 2013, n) JJA de 2013, o) SON de 2013 e p) DJF de 2013/2014.

bem úmidas sobre quase todo o Brasil, exceto na região sul onde o viés é positivo (Fig. 3).

No trimestre JFM de 2014, Reboita et al. (2015a) e Coelho et al. (2016) reportam o grande déficit de precipitação ocorrido no sudeste do Brasil. As Figs. 2 e 3 d, h, e, $\mathrm{p}, \mathrm{t}, \mathrm{x}$ mostram que somente a previsão do RegCPTECKF indicou viés negativo de precipitação sobre o sudeste do Brasil. Embora o RegCPTECKF tenha superestimado o déficit de precipitação, este foi o único a acertar o sinal do viés, pois todas as demais previsões superestimaram a pre- cipitação nesse trimestre (viés positivo). Em JFM, em geral, as previsões mostraram viés negativo de precipitação sobre a Amazônia e extremo sul do Brasil.

Para avaliar de forma mais precisa a qualidade das previsões, o viés da precipitação trimestral e o índice de concordância de Willmott $(d)$ foram calculados (Tabela 3) para os subdomínios mostrados na Fig. 1. Já na Fig. 4 são mostrados os valores médios trimestrais da precipitação prevista e observada. Nessa figura, também foi incluído o ensemble físico entre o RegCPTECKF e o 
Tabela 3 - Viés e índice de concordância de Willmott (d) das previsões de precipitação (mm/dia) comparadas aos dados do CPC e TRMM e de temperatura do ar a 2 metros $\left({ }^{\circ} \mathrm{C}\right)$ comparadas à reanálise ERA-Interim e CFSR.

\begin{tabular}{|c|c|c|c|c|c|c|c|c|c|}
\hline & & Viés CPC & Viés TRMM & d_preCPC & d_preTRMM & Viés ERA & Viés CFSR & d_tempERA & d_tempCFSR \\
\hline \multirow[t]{6}{*}{ AMZ } & СРТЕC & $-0,8$ & $-2,3$ & 0,90 & 0,76 & 0,1 & 0,2 & 0,29 & 0,57 \\
\hline & CFSv2 & $-0,6$ & $-2,2$ & 0,97 & 0,89 & $-0,1$ & 0,7 & 0,71 & 0,77 \\
\hline & RegCFSv2 & 0,6 & $-1,1$ & 0,91 & 0,88 & $-0,7$ & 0,5 & 0,28 & 0,26 \\
\hline & RegCPTECKF & $-0,6$ & $-2,2$ & 0,95 & 0,82 & 0,0 & 0,6 & 0,83 & 0,81 \\
\hline & RegCPTECEman & $-0,6$ & $-2,2$ & 0,95 & 0,80 & $-0,5$ & 0,1 & 0,57 & 0,93 \\
\hline & RegCPTECKFEm & $-0,6$ & $-2,2$ & 0,95 & 0,81 & $-0,3$ & 0,3 & 0,71 & 0,90 \\
\hline \multirow[t]{6}{*}{ NDE } & СРTEC & 3,1 & 2,9 & 0,36 & 0,42 & 0,0 & 0,8 & 0,89 & 0,73 \\
\hline & CFSv2 & 1,6 & 1,4 & 0,54 & 0,68 & $-2,0$ & $-1,2$ & 0,32 & 0,54 \\
\hline & RegCFSv2 & 2,3 & 2,1 & 0,44 & 0,58 & $-3,1$ & $-1,9$ & 0,17 & 0,33 \\
\hline & RegCPTECKF & 0,3 & 0,1 & 0,68 & 0,73 & 0,2 & 1,1 & 0,56 & 0,49 \\
\hline & RegCPTECEman & 0,2 & 0,0 & 0,86 & 0,84 & $-1,1$ & $-0,2$ & 0,50 & 0,88 \\
\hline & RegCPTECKFEm & 0,3 & 0,0 & 0,81 & 0,84 & $-0,4$ & 0,5 & 0,64 & 0,67 \\
\hline \multirow[t]{6}{*}{ SDE } & CPTEC & 1,1 & 0,7 & 0,71 & 0,86 & 0,3 & 2,2 & 0,95 & 0,62 \\
\hline & CFSv2 & 1,0 & 0,6 & 0,69 & 0,85 & $-2,6$ & $-0,5$ & 0,69 & 0,94 \\
\hline & RegCFSv2 & 0,7 & 0,2 & 0,71 & 0,85 & $-2,9$ & $-0,8$ & 0,65 & 0,88 \\
\hline & RegCPTECKF & $-1,2$ & $-1,7$ & 0,55 & 0,55 & $-0,1$ & 1,6 & 0,96 & 0,69 \\
\hline & RegCPTECEman & 0,7 & 0,2 & 0,75 & 0,87 & $-1,5$ & 0,4 & 0,81 & 0,91 \\
\hline & RegCPTECKFEm & $-0,3$ & $-0,7$ & 0,81 & 0,80 & $-0,8$ & 1,0 & 0,92 & 0,80 \\
\hline \multirow[t]{6}{*}{ SUL } & CPTEC & $-1,1$ & $-2,1$ & 0,59 & 0,53 & 1,8 & 2,6 & 0,93 & 0,81 \\
\hline & CFSv2 & 0,5 & $-0,6$ & 0,48 & 0,43 & $-1,3$ & 0,1 & 0,95 & 0,98 \\
\hline & RegCFSv2 & $-1,4$ & $-2,5$ & 0,48 & 0,50 & $-0,4$ & 0,5 & 0,99 & 0,99 \\
\hline & RegCPTECKF & $-1,7$ & $-2,7$ & 0,48 & 0,46 & 0,7 & 1,8 & 0,95 & 0,82 \\
\hline & RegCPTECEman & $-1,0$ & $-2,0$ & 0,68 & 0,57 & $-0,1$ & 1,0 & 0,97 & 0,90 \\
\hline & RegCPTECKFEm & $-1,4$ & $-2,4$ & 0,57 & 0,51 & 0,3 & 1,4 & 0,97 & 0,86 \\
\hline
\end{tabular}

RegCPTECEman, que foi chamado de RegCPTECKFEm.

Primeiramente enfatiza-se que as próprias observações apresentam diferenças entre elas, sendo o TRMM mais chuvoso do que o CPC. De forma geral, a previsão da precipitação é melhor nas regiões $\mathrm{AMZ}$ e SDE, onde os modelos conseguem reproduzir o padrão do ciclo anual das observações e também com valores próximos aos observados. Considerando a região AMZ (Fig. 4a), as melhores previsões em termos de representação do ciclo anual foram obtidas com o RegCPTECKF, RegCPTECEman e CPTEC, porém estes subestimaram a precipitação em aproximadamente $2 \mathrm{~mm} /$ dia nos trimestres OND e nos seguintes em relação ao CPC. Estes trimestres onde tais previsões subestimam a precipitação, são melhores representados pelo CFSv2. De forma geral, todas as previsões tiverem $d \geq 0,9$. Na região NDE (Fig. 4b), a precipitação é melhor prevista pelo RegCPTECEman em todos os trimestres que também mostra o melhor valor do índice de concordância $(d=0,84)$. As outras previsões mostram bons resultados somente entre MJJ e ASO; pois no restante do ano superestimam a precipitação (exceto RegCPTECKF, que a subestima). Na região SDE (Fig. 4c), os modelos conseguem reproduzir bem o padrão do ciclo anual de FMA a ASO, embora com subestimativas. Já nos demais trimestres, as previsões deslocam o máximo de precipitação que ocorre entre OND e DJF: algumas antecipam (RegCPTECKF e RegCPTECEman) e outras atrasam a ocorrência do máximo (CFSv2 e RegCFSv2). Nessa região, o índice de concordância foi acima de 0,7 , exceto na previsão RegCPTECKF. O RegCPTECEman teve $d=0,75$ em relação ao CPC e 0,85 em relação ao TRMM. Dessa forma, se fosse necessário escolher uma configuração de modelo para o SDE, o RegCPTECEman poderia ser uma opção. Na região SUL (Fig. 4d), além dos modelos não acompanharem o padrão das observações, também as subestimam. Somente o CFSv2 é que mostra superestimativas em relação ao CPC e também com desvios mais próximo dessa observação do que os modelos que subestimam a precipitação. Embora em termos de padrão e intensidade da precipitação o CFSv2 mostra-se mais próximo das observações, a variabilidade trimestral é melhor capturada pela previsão RegCPTECEman, pois o índice de concordância dessa é $d=0,68$ em relação ao CPC enquanto o do CFSv2 é $d=0,48$. As condições mais secas do RegCFSv2 em rela- 

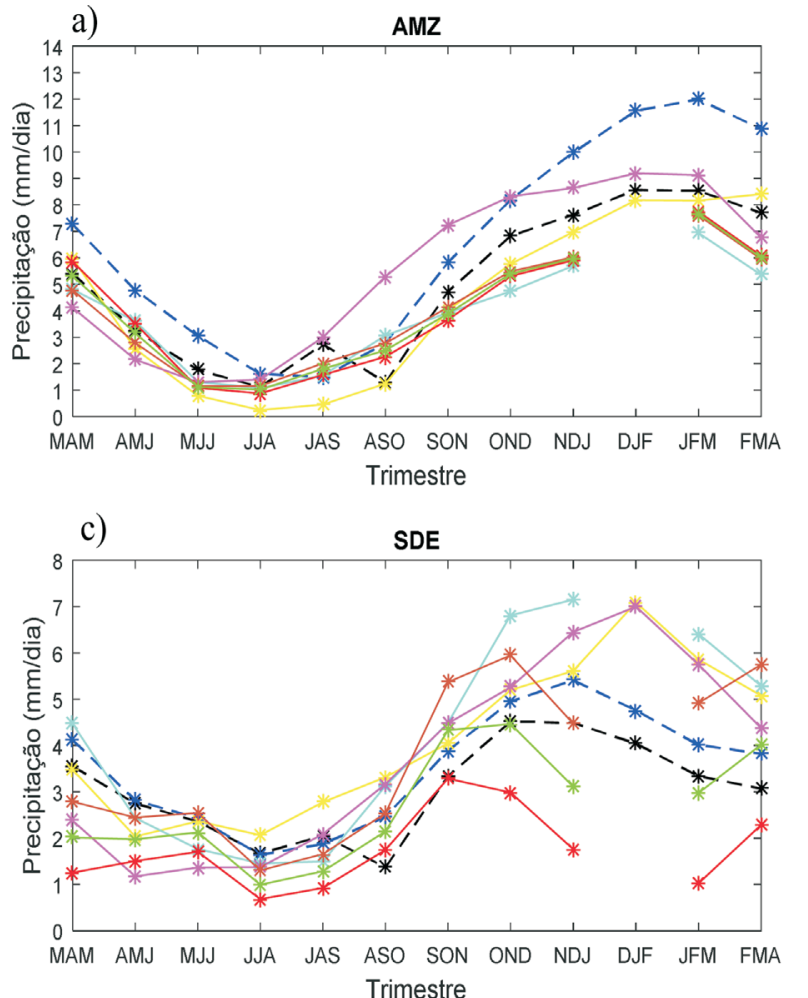
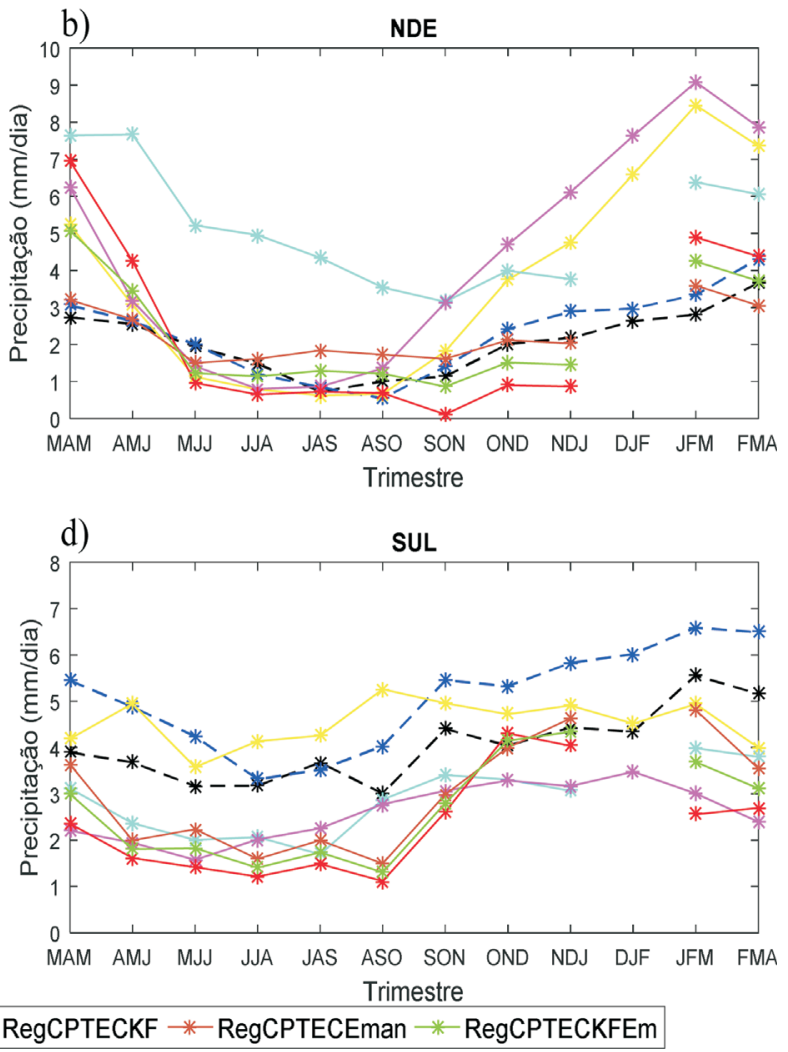

Figura 4 - Médias sazonais da precipitação (mm/dia) nos subdomínios apresentados na Fig. 1. A linha preta representa o CPC, a azul o TRMM, a amarela o CFSv2, a ciano o CPTEC, a rosa o RegCFSv2, a vermelha o RegCPTECKF, a marrom o RegCPTECKF e a verde o RegCPTECKFEm.

ção ao CFSv2 no SUL também foi um resultado obtido por Gonçalves (2015), que aninharam o CFSv2 ao RegCM.

Com relação à temperatura do ar a 2 metros de altura (Figs. 5 e 6), enquanto as previsões RegCPTECKF e RegCPTECEman (Fig. 5) mostram habilidade em reduzir o viés quente do modelo do CPTEC sobre o sudeste da AS, as previsões do RegCFSv2 (Fig. 6) aumentam o viés frio do CFSv2, principalmente no nordeste e sudeste do Brasil. Em MAM (Figs. 5 e 6 a, e, i, m, q, u), o RegCPTECKF e o CPTEC (Fig. 5) previram temperaturas similares às da ERA-Interim na região norte do Brasil, mas subestimaram as temperaturas no nordeste e superestimaram nas demais regiões do país e nas cercanias do Paraguai. Já, o RegCPTECEman foi mais similar às reanálises, mesmo assim, simulou viés frio em todo o setor leste do Brasil comparado a ERA-Interim e viés quente sobre o sudeste comparado ao CFSR. O RegCFSv2 e o CFSv2 (Fig. 6) subestimaram as temperaturas em todo o país exceto no estado do Rio Grande do Sul. De forma geral, em MAM, a melhor previsão de temperatura do ar sobre o Brasil foi a do CFSv2 comparado ao CFSR.

Em JJA, as previsões RegCPTECKF e RegCPTECEman (Fig. 5) reduziram o viés frio do CPTEC sobre o norte do Brasil, porém a RegCPTECKF previu temperaturas mais quentes no centro-oeste do país e sudeste da AS do que o CPTEC. O viés quente foi menor no
RegCPTECEman comparado ao RegCPTECKF. O RegCFSv2 e o CFSv2 (Fig. 6) apresentaram previsões similares, exceto pelo RegCFSv2 mostrar viés quente sobre os arredores de Rondônia e o CFSv2 sobre o estado do Amazonas. Nos demais trimestres (SON e JFM), nota-se que a RegCPTECKF e RegCPTECEman (Fig. 5) reduzem o viés quente do CPTEC sobre a região centro-oeste do Brasil e sudeste da AS, enquanto o RegCFSv2 (Fig. 6) intensifica o viés frio obtido pelo CFSv2. No trimestre JFM, nas regiões nordeste e sudeste do Brasil onde ocorreram vieses positivos de precipitação no $\mathrm{CFSv} 2$ e no RegCFSv2, na temperatura do ar encontram-se os vieses mais negativos desse período. Fato que é termodinamicamente esperado, pois se há precipitação, a energia é utilizada para a evaporação da água ao invés do aquecimento da atmosfera via calor sensível. A melhoria do RegCFSv2 ocorre sobre o sul do Brasil, onde há redução do viés frio. Em todos os períodos analisados, de forma geral, a melhor previsão de temperatura sobre o país foi a do RegCPTECEman.

A boa performance das previsões de temperatura pelo RegCPTECKF e RegCPTECEman também é mostrada através das médias computadas nos subdomínios indicados na Fig. 1 e comparadas com as da reanálise ERA-Interim e CFSR (Fig. 7) e pelo índice de concordância de Willmott (Tabela 3). Na região AMZ (Fig. 7a), o RegCPTECKF, 
a)

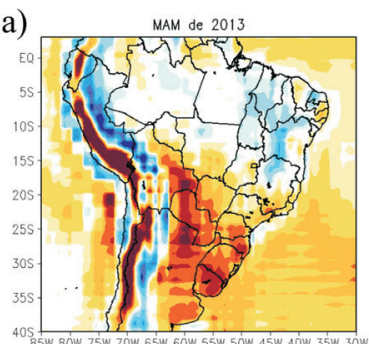

e)
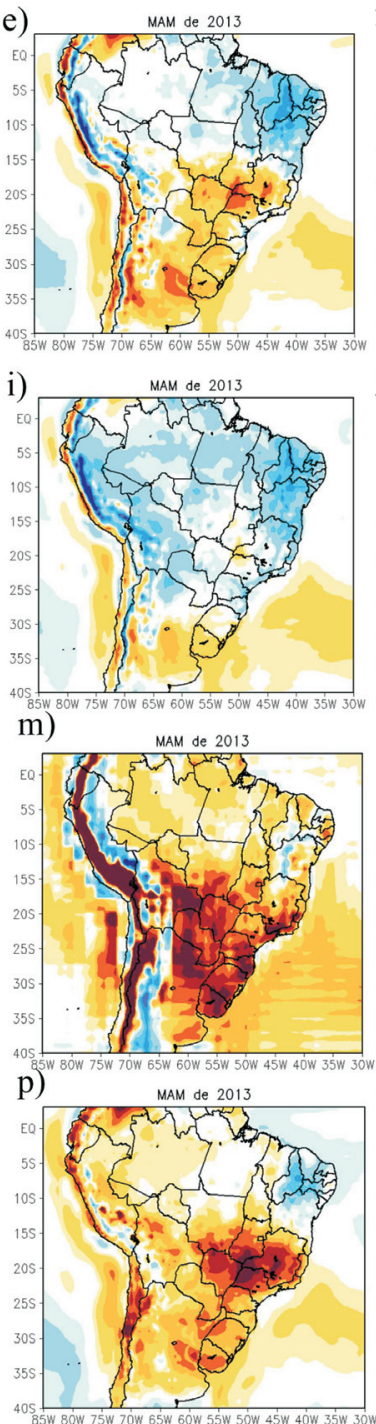

s)

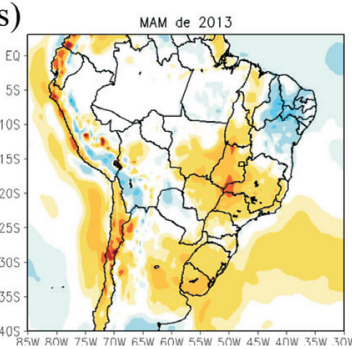

b)

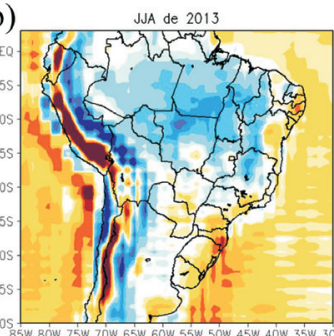

f)

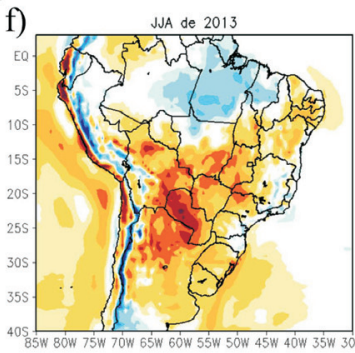

j)

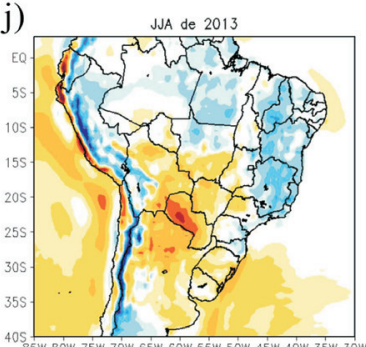

n)
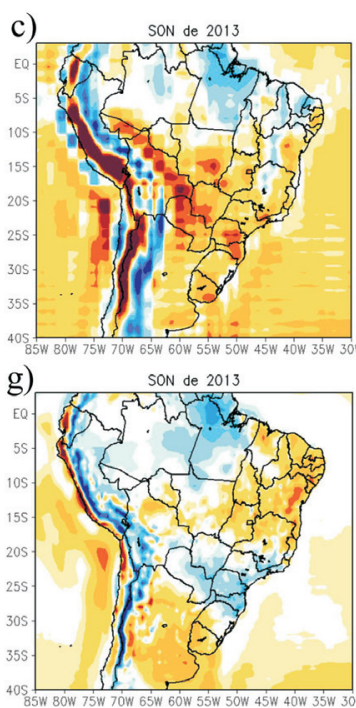

k)

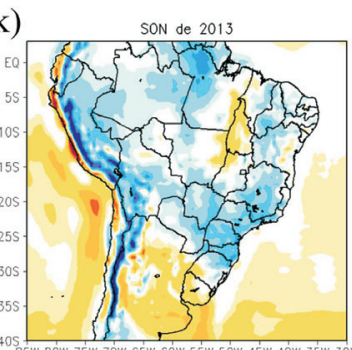

o)
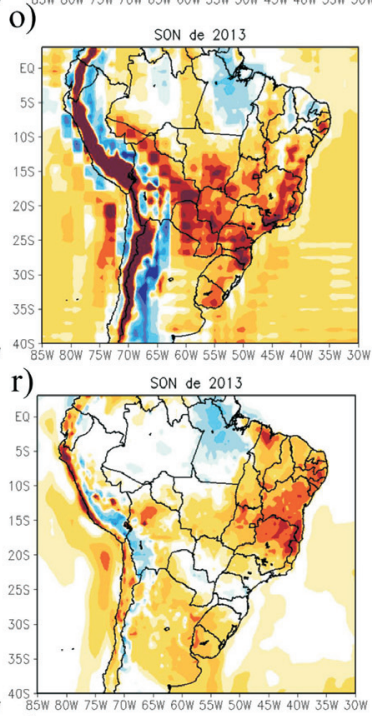

t)
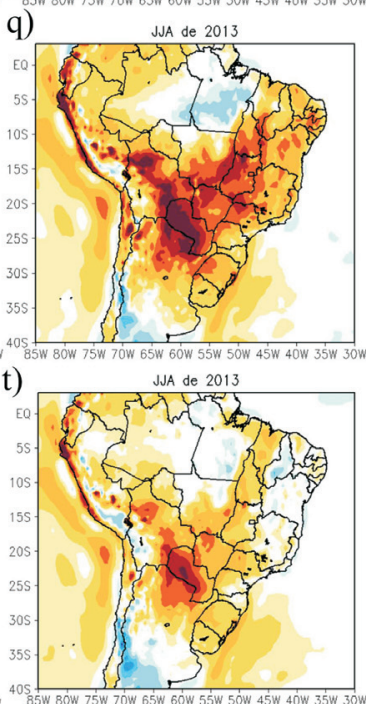

u)

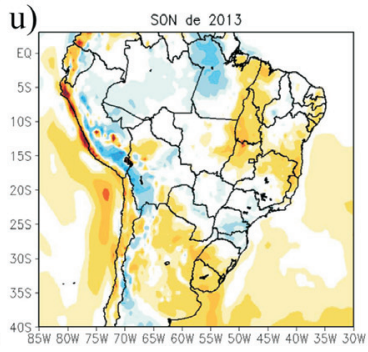

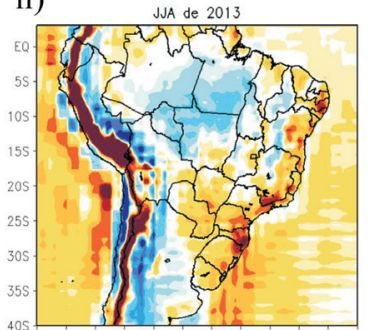

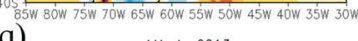

d)

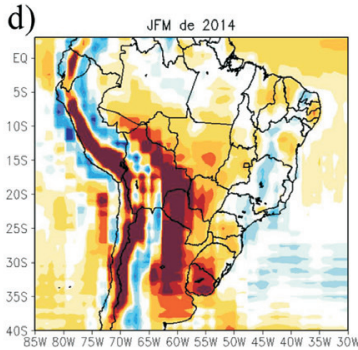

h)

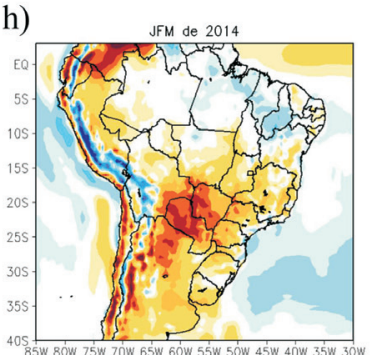

1)

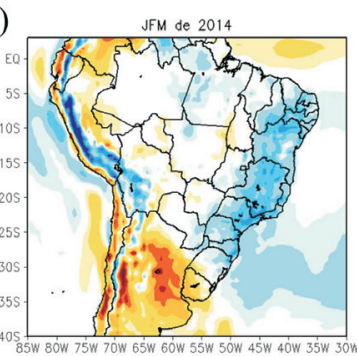

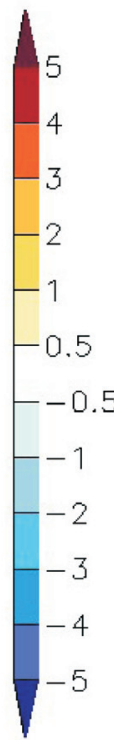

Figura 5 - Viés da temperatura do ar a 2 metros $\left({ }^{\circ} \mathrm{C}\right.$ ). CPTEC menos ERA-Interim (primeira linha): a) MAM de 2013, b) JJA de 2013 , c) SON de 2013 e d) JFM de 2014. RegCPTECKF menos ERA-Interim (segunda linha): e) MAM de 2013, f) JJA de 2013, g) SON de 2013 e h) JFM de 2014. RegCPTECEman menos ERA-Interim (terceira linha): i) MAM de 2013, j) JJA de 2013, k) SON de 2013 e 1) JFM de 2014. CPTEC menos CFSR (quarta linha): m) MAM de 2013, n) JJA de 2013 e o) SON de 2013. RegCPTECKF menos CFSR (quinta linha): p) MAM de 2013, q) JJA de 2013 e r) SON de 2013. RegCPTECEman menos CFSR (sexta linha): s) MAM de 2013, t) JJA de 2013, u) SON de 2013. 

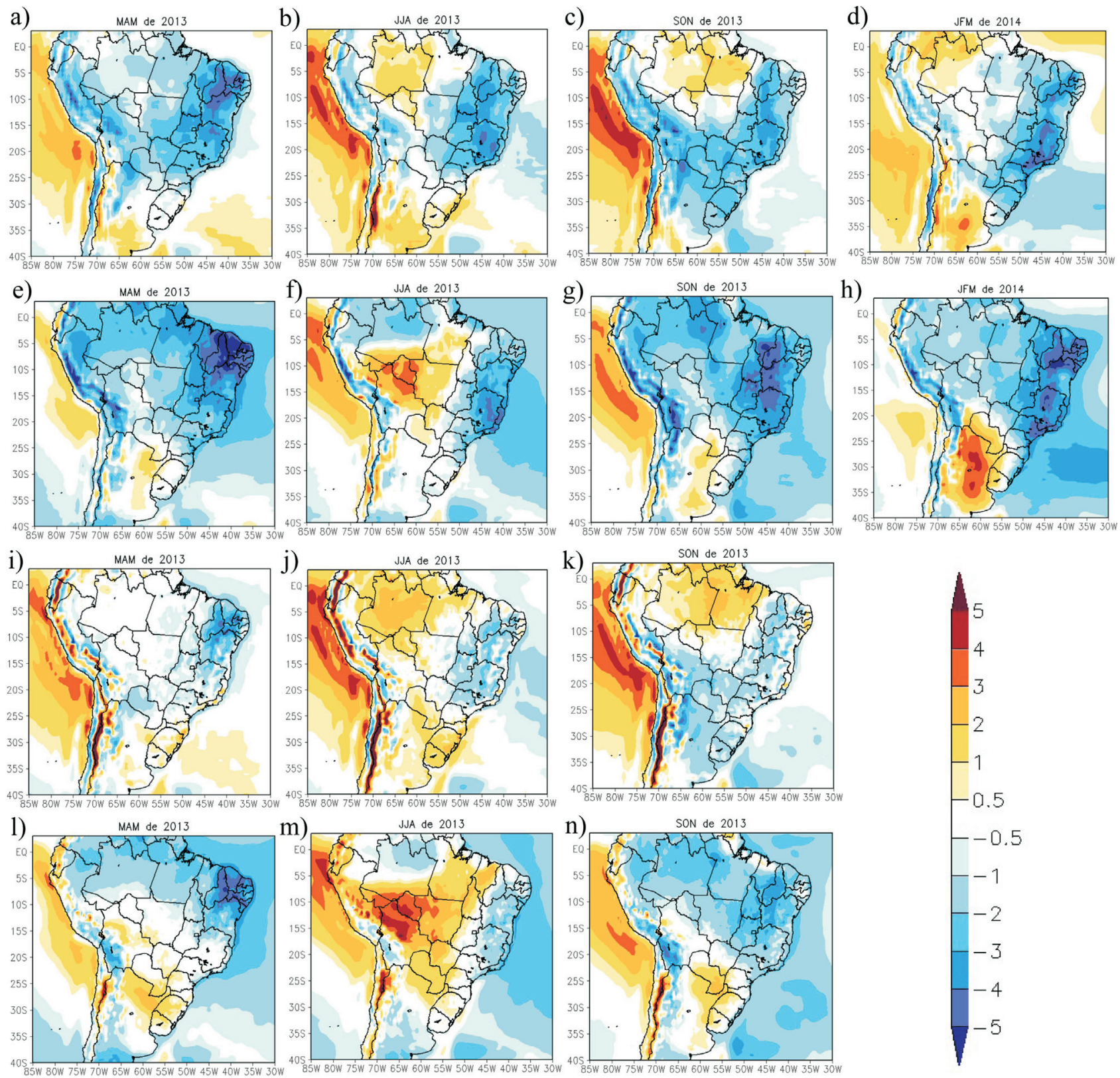

Figura 6 - Viés da temperatura do ar a 2 metros $\left({ }^{\circ} \mathrm{C}\right)$ : CFSv2 menos Era-Interim (primeira linha): a) MAM de 2013, e) JJA de 2013, i) SON de 2013 e m) DJF de 2013/2014. RegCFSv2 menos Era-Interim (segunda linha): b) MAM de 2013, f) JJA de 2013, j) SON de 2013 e n) DJF de 2013/2014. CFSv2 menos CFSR (terceira linha): c) MAM de 2013, g) JJA de 2013 e k) SON de 2013. RegCFSv2 menos CFSR (quarta linha): d) MAM de 2013, h) JJA de 2013 e l) SON de 2013.

RegCPTECEman, o ensemble destes dois e o CFSv2 possuem médias próximas aos valores observados, enquanto o CPTEC e RegCFSv2 mostram vieses maiores. Além disso, esses dois últimos estão fora de fase entre si: enquanto um mostra superestimava o outro subestimativa. $\mathrm{O}$ RegCPTECKF tem índice de concordância $0,83(0,81) \mathrm{em}$ relação à reanálise ERA-Interim (CFSR), enquanto o RegCPTECEman, 0,57 (0,93), respectivamente. No NDE (Fig. 7b), a melhor previsão de temperatura é obtida pelo CPTEC comparado à ERA-Interim (com $d=0,89)$ e pelo RegCPTECEman comparado ao CFSR (com $d=0,88$ ). O
CFSv2 e RegCFSv2 subestimam as temperaturas nessa região e o RegCPTECKF superestima de JJA até FMA. O SDE (Fig. 7c) é a região em que as temperaturas das reanálises mais se diferenciam (chegando a cerca de $3{ }^{\circ} \mathrm{C}$ ). Entre MAM a OND, as previsões do RegCPTECKF, RegCPTECEman e o ensemble destes dois mostram valores entre as duas reanálises, porém com um mínimo em MJJ que os diferencia das reanálises. Por outro lado, embora o CFSv2 e o RegCFSv2 subestimem as temperaturas, eles reproduzem melhor o padrão do ciclo anual. Por esse motivo, que o índice de concordância do CFSv2 em relação ao 


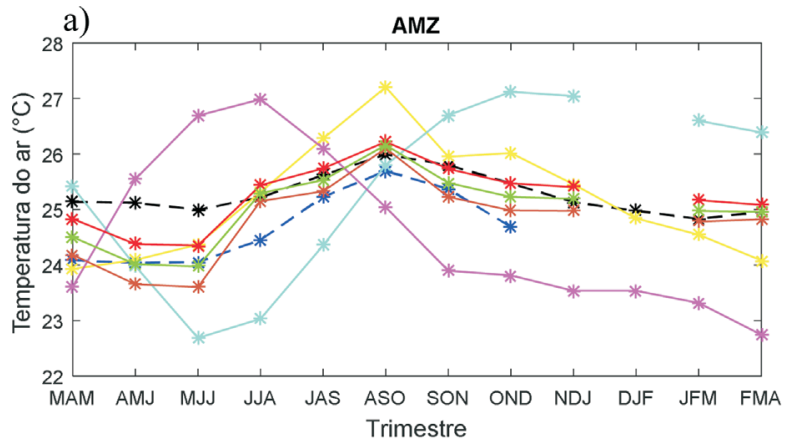

c)

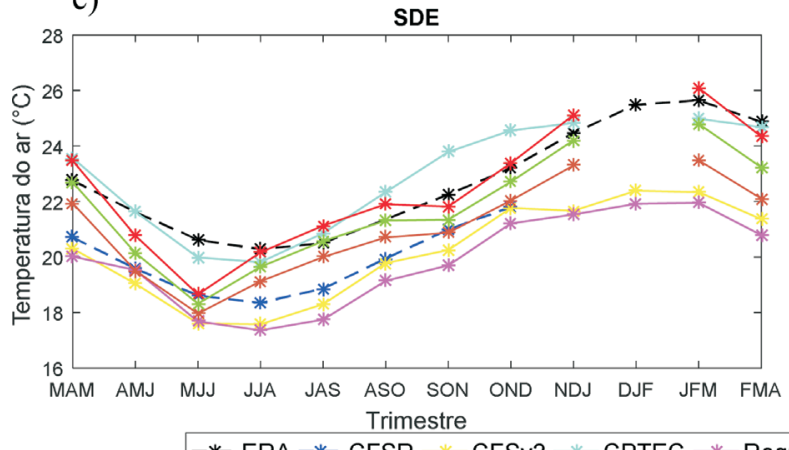

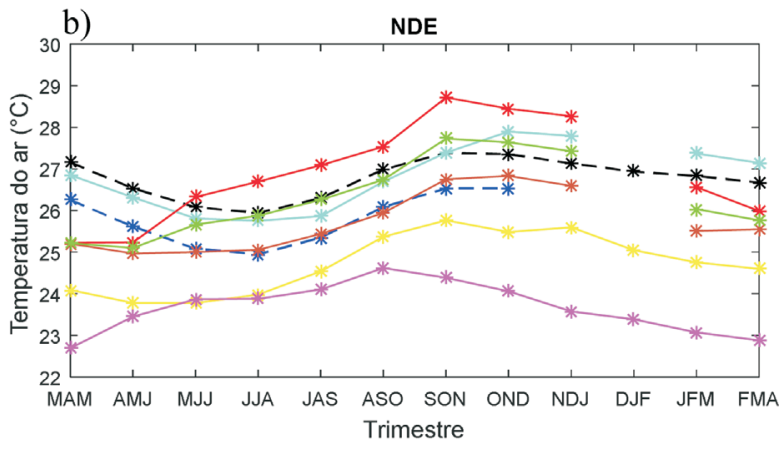

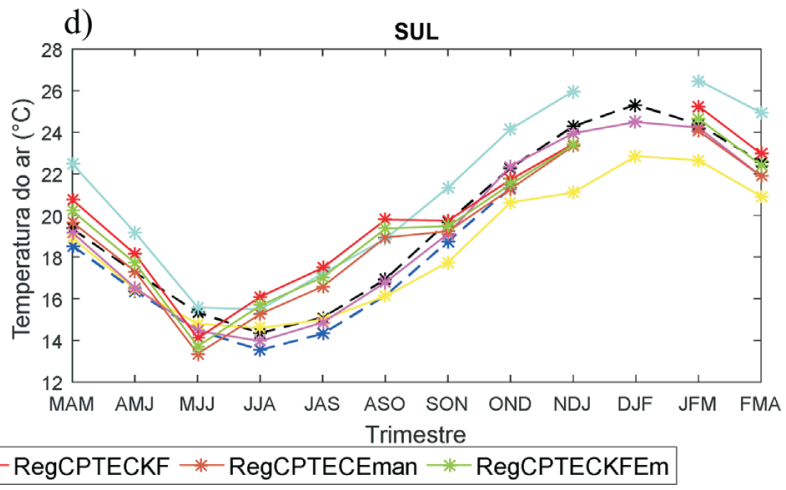

Figura 7 - Médias sazonais da temperatura do $\operatorname{ar}\left({ }^{\circ} \mathrm{C}\right)$. A linha preta representa a reanálise ERA-Interim, a azul a reanálise CFSR, a amarela o CFSv2 (previsão), a ciano o CPTEC, a rosa o RegCFSv2, a vermelha o RegCPTECKF, a marrom o RegCPTECEman e a verde o RegCPTECKFEm.

CFSR é alto (0,94). No SUL (Fig. 7d), de forma geral, as previsões mostram pouca diferença em valores em relação às reanálises. Porém, a previsão do RegCFSv2 é a que representa perfeitamente o ciclo anual, tanto em padrão quando em valores. Esse resultado também se reflete no índice de concordância $(0,99$ em relação as duas reanálises). Nessa região, o RegCPTECKF, RegCPTECEman e o ensemble destes também mostram um mínimo em MJJ que não é registrado nas reanálises.

\subsection{O verão de 2014}

Os trimestres DJF e JFM foram atípicos sobre o sudeste do Brasil no ano de 2014 (Reboita et al., 2015a; Coelho et al., 2016), devido à escassez de precipitação e elevadas temperaturas. Na seção anterior foi mencionado que apenas o RegCPTECKF conseguiu mostrar viés seco sobre o sudeste do Brasil nesse período, enquanto as demais previsões superestimaram a precipitação (Figs. 2 e 3 ). Aqui se tem, portanto, uma justificativa para investimentos em pesquisas de previsão sazonal do clima, uma vez que, as políticas públicas a serem empregadas em muitos setores são dependentes do que acontece na atmosfera em diferentes escalas temporais, como a sazonal.

Reboita et al. (2015b) compararam os padrões da circulação atmosférica previstos pelo CFSv2 e RegCFSv2 com o registrado na ERA-Interim a fim de mostrar o que conduziu ao viés positivo de precipitação nos modelos. Estudos prévios como o de Nogués-Peagle e Mo (1997) têm relacionado os verões secos no sudeste do Brasil com a supressão da Zona de Convergência do Atlântico Sul (ZCAS). Nessas situações, o Anticiclone Subtropical do Atlântico Sul (ASAS) aparece deslocado para oeste em relação a sua posição climatológica e o setor oeste do cavado do nordeste do Brasil permanece sobre parte do sudeste e nordeste do Brasil, sendo configurações que dificultam a organização de precipitação. De fato, essas características foram observadas em JFM de 2014 inibindo a precipitação no sudeste do país de acordo com a reanálise ERA-Interim. Entretanto, essas características não aparecem na previsão. A Fig. $8 \mathrm{~b}$ mostra na reanálise parte do ASAS sobre o sudeste do Brasil se assemelhando ao padrão climatológico de inverno (Reboita et al., 2010), enquanto que o CFSv2 e RegCFSv2 simulam um anticiclone muito mais fraco (Fig. 8a, d). A diferença computada entre as previsões e a reanálise mostra altura geopotencial em $1000 \mathrm{hPa}$ de cerca de $20 \mathrm{~m}$ mais baixa nas previsões sobre o sudeste do Brasil e oceano Atlântico Sul (Fig. 8c, f).

Em altos níveis (Fig. 9), na reanálise aparece o cavado do nordeste do Brasil com orientação nordeste-sudoeste e com seu setor oeste sobre as regiões nordeste e sudeste do país (Fig. 9b). Já nas previsões (Fig. 9 a,d), o sistema aparece deslocado sobre o oceano Atlântico e com orientação noroeste-sudeste. Na reanálise, a configuração do cavado atua de forma a inibir a organização de precipitação sobre o sudeste do Brasil, o que não ocorre pela configuração desse sistema nas previsões; logo, nestas há mais chuva. A temperatura do ar geralmente é modulada pela 
precipitação, se há (não há) ocorrência de chuva esperamse menores (maiores) temperaturas. Dessa forma, os modelos responderam ao padrão obtido na precipitação. Para sumarizar, a baixa performance dos modelos em prever a precipitação em JFM esteve associada com a fraca habilidade de simular os padrões atmosféricos observados.
CFSv2

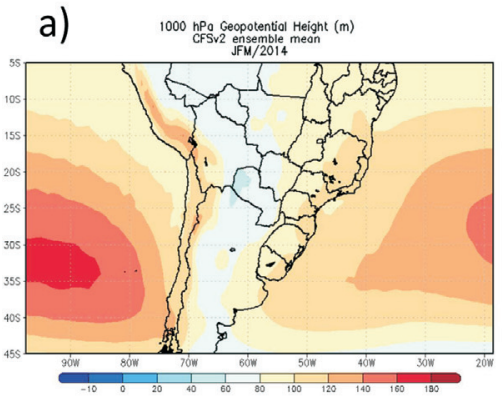

RegCFSv2

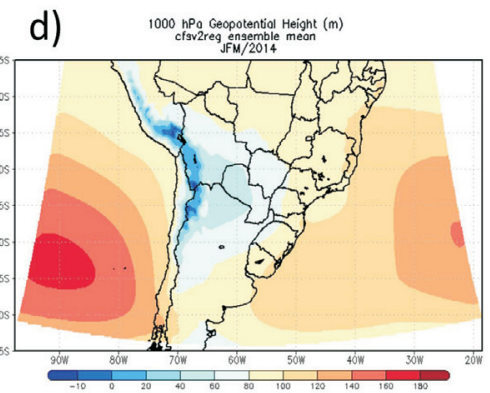

ERA-Interim

b)

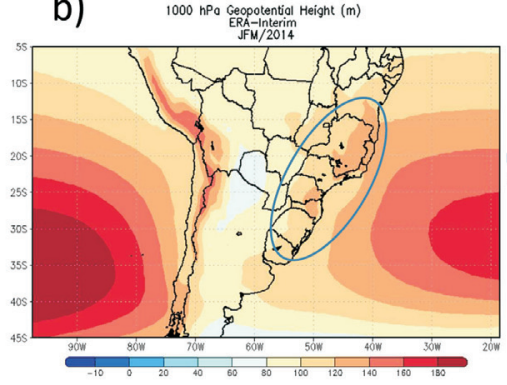

ERA-Interim

e)

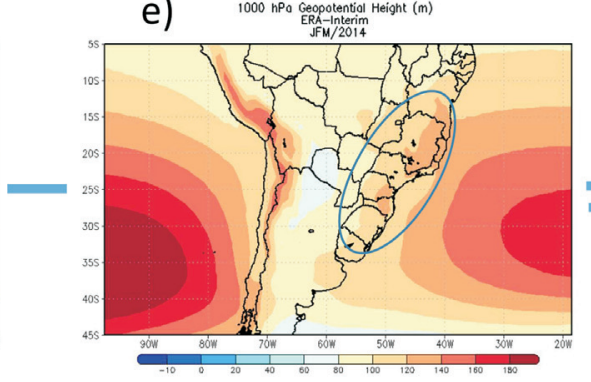

CFSV2 - ERA

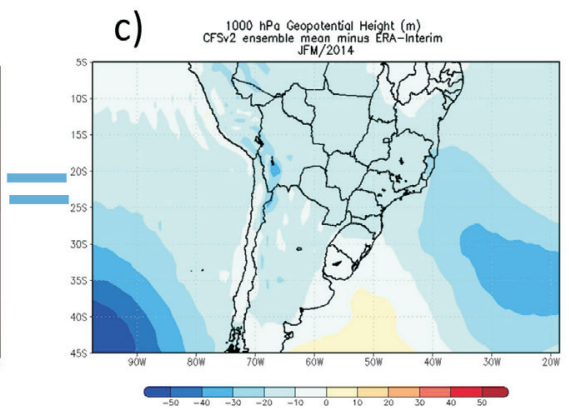

RegCFSv2 - ERA

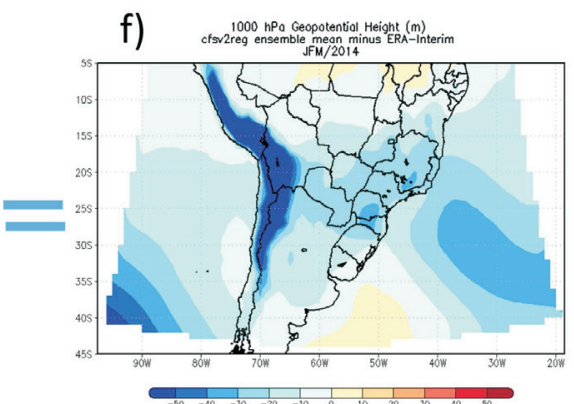

Figura 8 - Altura geopotencial (m) em 1000 hPa: a) CFSv2, b) ERA-Interim, c) diferença (a)-(b), d) RegCFSv2, e) ERA-Interim e (f) diferença (d)-(f). Nas figuras (b) e (e), a elipse em azul indica a região com maior pressão atmosférica na reanálise em comparação com as previsões.

CFSv2

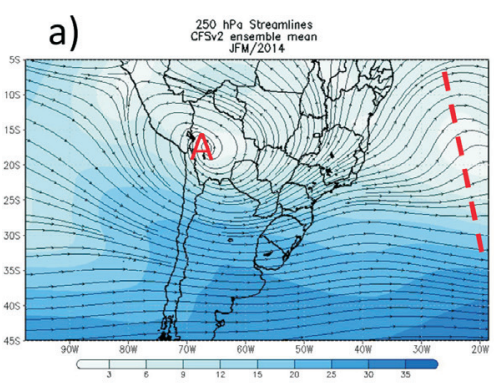

ERA-Interim

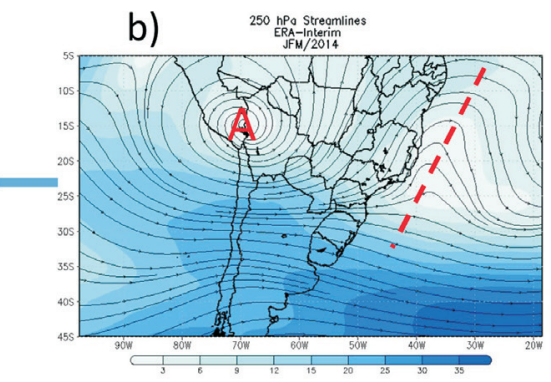

CFSv2 - ERA

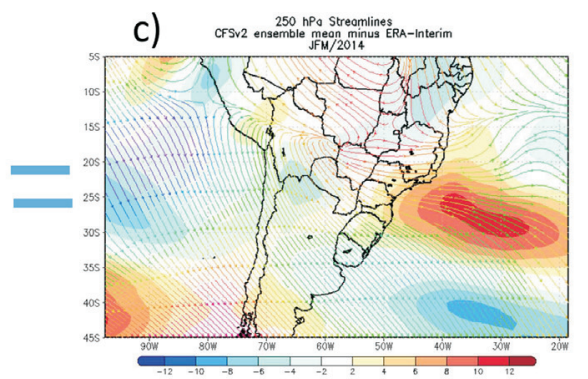

\section{RegCFSv2}

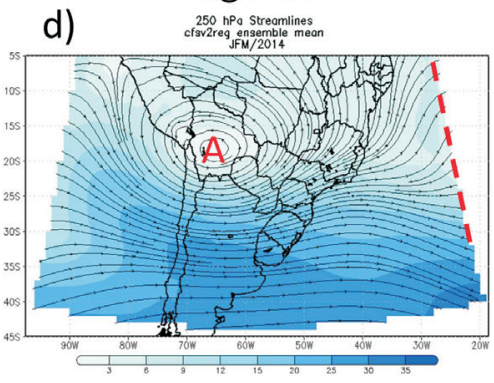

ERA-Interim

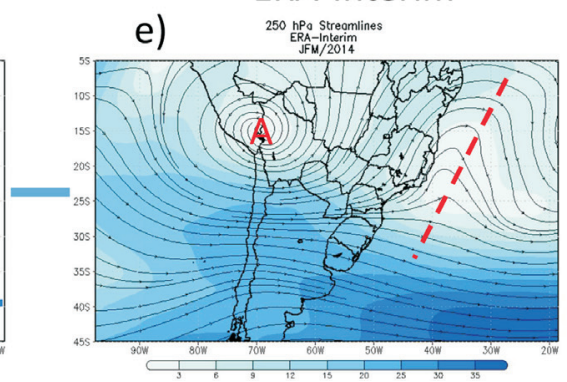

RegCFSv2 - ERA

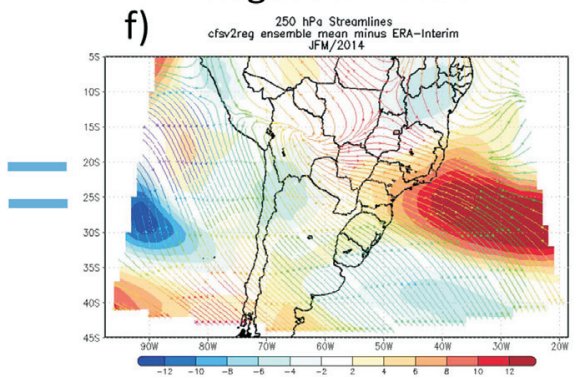

Figura 9 - Linhas de corrente e intensidade do vento (m/s) em 250 hPa: a) CFSv2, b) ERA-Interim, c) diferença (a)-(b), d) RegCFSv2, e) ERA-Interim e (f) diferença (d)-(f). Nas figuras a letra A indica a posição da alta da Bolívia, enquanto a linha vermelha tracejada, a orientação do cavado do nordeste do Brasil. 


\subsection{Períodos secos e úmidos}

Na Tabela 4 têm-se o número total de períodos secos e úmidos identificados nos quatro domínios em estudo e considerando todos os trimestres (MAM a FMA). Destaca-se que devido à ausência da previsão de outubro de 2013 do modelo do CPTEC, que seria referente à previsão de DJF, tal trimestre foi excluído da análise, para que se tenha 11 trimestres previstos em todos os dados.

De forma geral, o TRMM apresenta menor número de períodos secos e úmidos do que o $\mathrm{CPC}$; a exceção ocorre na região SUL (Tabela 4), quando o TRMM detecta quatro eventos úmidos enquanto o CPC nenhum. Em todos os subdomínios, as previsões do CFSv2 e do RegCFSv2 superestimam o número de períodos secos e úmidos observados. Já o CPTEC, RegCPTECKF e RegCPTECEman apresentam menor número de eventos do que essas previsões, mas também, de maneira geral, superestimam o número de períodos secos e úmidos. Na AMZ, a previsão do CPTEC é a mais próxima do CPC; nas regiões NDE e SUL a previsão do RegCPTECKF é mais similar ao TRMM e na região sudeste a previsão do RegCPTECKF é a mais próxima do CPC.

\section{Conclusões}

O presente estudo verificou a qualidade das previsões sazonais de precipitação e temperatura do ar sobre o Brasil de dois modelos climáticos globais (CFSv2 e CPTEC) e de um modelo regional (RegCM4). Além disso, foi verificado se o RegCM4 adiciona valor às previsões sazonais dos modelos globais. Foram utilizadas duas versões do RegCM: a 4.3 e a 4.5. O RegCM4.3 foi dirigido por seis membros do CFSv2 e as simulações denominadas de RegCFSv2. Como ao longo do estudo foi liberada uma versão mais recente do modelo regional, o RegCM4.5, e contando com um novo esquema de convecção cumulus, Kain Fritsch, utilizou-se essa versão e esquema para realizar previsões dirigidas por um membro do modelo global do CPTEC. Entretanto, também se realizaram previsões com o RegCM4.5 e o esquema de Emanuel dirigidas pelo modelo do CPTEC. Essas foram denominadas de RegCPTECKF e RegCPTECEman, respectivamente. Todas as previsões iniciaram cerca de dois meses antes do trimestre de interesse. Foram realizadas 72 simulações dirigindo o modelo regional RegCM com o CFSv2 e 22 com o CPTEC, sendo 11 com convecção cumulus de Kain Fritsch e 11 com a convecção cumulus de Emanuel.

Realizando a validação das previsões sazonais por regiões do Brasil (Tabela 5), verificou-se que na região amazônica tanto a precipitação quanto a temperatura do ar foram melhores previstas pelo RegCPTECKF e RegCPTECEman; no nordeste, novamente os melhores resultados para ambas as variáveis foi com o RegCPTECEman; na região sudeste, a precipitação foi bem representada pelo RegCPTECEman, enquanto que a temperatura do ar foi prevista adequadamente em todas as previsões; mas com melhor representação da fase do ciclo anual pelo CFSv2. Já na região sul do país, a precipitação foi melhor prevista pelo sistema CFSv2 e a temperatura do ar pelo RegCFSv2. Esse resultado para a região sul do

Tabela 4 - Quantidade de períodos secos e úmidos previstos e observados nos trimestres de MAM/2013 a FMA/2014, exceto o trimestre de DJF, nas regiões Amazônica (AMZ), Nordeste (NDE), Sudeste (SDE) e Sul do Brasil (SUL).

\begin{tabular}{|c|c|c|c|c|c|c|c|c|}
\hline & & \multicolumn{2}{|c|}{ Globais } & \multirow{2}{*}{$\begin{array}{c}\text { Ensemble } \text { RegCM4.3 } \\
\text { RegCFSv2 }\end{array}$} & \multicolumn{2}{|c|}{ RegCM4.5 } & \multicolumn{2}{|c|}{ Observados } \\
\hline & & CFSv2 & CPTEC & & RegCPTECKF & RegCPTECEman & $\mathrm{CPC}$ & TRMM \\
\hline \multirow[t]{2}{*}{ AMZ } & Úmido & 8 & 5 & 10 & 6 & 7 & 4 & 3 \\
\hline & Seco & 6 & 5 & 10 & 7 & 11 & 2 & 2 \\
\hline \multirow[t]{2}{*}{$\mathrm{NDE}$} & Úmido & 10 & 8 & 11 & 8 & 8 & 9 & 7 \\
\hline & Seco & 10 & 8 & 11 & 3 & 9 & 5 & 3 \\
\hline \multirow[t]{2}{*}{ SDE } & Úmido & 5 & 8 & 9 & 7 & 6 & 4 & 1 \\
\hline & Seco & 11 & 9 & 9 & 1 & 7 & 4 & 3 \\
\hline \multirow[t]{2}{*}{ SUL } & Úmido & 4 & 6 & 4 & 3 & 2 & 0 & 4 \\
\hline & Seco & 6 & 5 & 8 & 0 & 3 & 0 & 0 \\
\hline
\end{tabular}

Tabela 5 - Síntese das melhores previsões sazonais obtidas nos subdomínios de estudo.

\begin{tabular}{lll}
\hline Regiões do Brasil & Precipitação & Temperatura do ar \\
\hline Amazônia & RegCPTECKF/RegCPTECEman/CPTEC & RegCPTECKF/RegCPTECEman \\
Nordeste & RegCPTECEman & CPTEC/RegCPTECEman \\
Sudeste & RegCPTECEman & CFSv2 (por causa do padrão do ciclo anual) \\
Sul & CFSv2/RegCPTECEman & RegCFSv2 \\
\hline
\end{tabular}


Brasil é consistente com o de Gonçalves (2015) que mostrou que o RegCM4 dirigido pelo CFSv2 tem boa performance em tal região.

De forma geral, o presente estudo indica que o modelo regional adiciona valor às previsões dos modelos globais. Entretanto, também é necessário desatacar se o RegCM4 aninhado às simulações do modelo do CPTEC produz resultados consistentes com a sua versão prévia, RegCM3, que foi aninhada às saídas do modelo do CPTEC-COLA por Machado e da Rocha (2011). A comparação dos resultados de ambos os trabalhos mostrou que na região nordeste do Brasil as duas versões do RegCM reduzem o viés úmido do CPTEC produzindo previsões mais similares aos valores observados. Nessa região, o RegCM3 subestimava a temperatura do ar, enquanto o RegCM4 passa a superestimar essa variável. Já a versão mais recente do modelo do CPTEC produz previsões de temperatura do ar mais similares aos valores observados. $\mathrm{Na}$ região sudeste do Brasil, o RegCM4 continua com a tendência do RegCM3 de subestimar a precipitação comparado ao CPTEC e observações. Entretanto, o RegCM4 corrige o viés frio da versão 3 produzindo previsões próximas às observações. Na região sul, ambas as versões do RegCM apresentam resultados similares e com algumas deficiências na previsão da precipitação e temperatura. De forma geral, como o RegCM4 melhorou a previsão da temperatura do ar na região sudeste do Brasil e não mostrou deficiências a mais do que na sua versão prévia, conclui-se que adicionou valor em relação à versão anterior.

O verão de 2014 foi atípico na região sudeste do Brasil, isto é, anomalamente seco e quente (Reboita et al., 2015a; Coelho et al., 2016), o que gerou graves consequências negativas à população devido à falta de água (Marengo et al., 2015). Nem os modelos globais em estudo e nem o RegCFSv2 foi capaz de prever essa condição. Reboita et al. (2015b) mostraram que o erro na previsão da circulação atmosférica em baixos e altos níveis conduziu à previsão errônea da temperatura do ar e precipitação no CFSv2 e RegCFSv2. Um estudo adicional da circulação atmosférica prevista pelo modelo regional dirigido com as saídas do modelo global do CPTEC está sendo realizado e será apresentado em outro artigo.

Embora estudos adicionais que avaliem o desempenho das previsões sazonais do RegCM4 com diferentes esquemas de parametrização de convecção cumulus e de interação superfície-atmosfera ainda sejam necessários, sugere-se que o RegCPTECEman seja colocado em modo operacional para que num primeiro momento suas previsões possam continuar sendo validadas e, na sequência, que essa combinação de modelos possa contribuir com os prognósticos elaborados rotineiramente pelo CPTEC, Instituto Nacional e Meteorologia e Grupo de Estudos Climáticos da Universidade de São Paulo. Além disso, se houver recursos computacionais disponíveis o suficiente (tempo de processamento e espaço em disco), sugere-se a realização de ensembles temporais com 0 RegCPTECEman, RegCPTECKF e RegCFSv2.

\section{Referências}

BLUNDEN, J.; ARNDT, D.S. Eds. State of the Climate in 2013. Bulletin of the American Meteorological Society, v. 95, n. 7, S1-S257, 2014.

CAVALCANTI, I.F.A.; MARENGO, J.A.; SATYAMURTY, P.; NOBRE, C.A.; TROSNIKOV, I. et al. Global Climatological Features in a Simulation Using the CPTEC AGCM. Journal of Climate, v. 15, n. 21, p. 2965-2988, 2002.

CHEN, M.; SHI, W.; XIE, P.; SILVA, V.B.S.; KOUSKY, V.E.; WAYNE HIGGINS, R.; JANOWIAK, J.E. Assessing objective techniques for gauge-based analyses of global daily precipitation. Journal of Geophysical Research, v. 113, D04110, p. 1-13, 2008.

CHOU, S.C.; NUNES, A.M.B.; CAVALCANTI, I.F.A. Extended range forecasts over South America using the regional Eta Model. Journal of Geophysical Research, v. 105, D8, p. 10147-10160, 2000.

CHOU, S.C.; BUSTAMANTE, J.F.; GOMES, J.L. Evaluation of Eta Model seasonal precipitation forecasts over South America. Nonlinear Processes in Geophysics, v. 12, p. 537-555, 2005.

COELHO, C.A.S., CAVALCANTI, I.A.F.; COSTA, S.M.S.; FREITAS, S.R.; ITO, E.R.; LUZ, G.; SANTOS, A.F.; NOBRE, C.A.; MARENGO, J.A; PEZZA, A.B. Climate diagnostics of three major drought events in the Amazon and illustrations of their seasonal precipitation predictions. Meteorological Applications, v. 19, p. 237-255, 2012.

COELHO, C.A.S.; OLIVEIRA; C.P.; AMBRIZZI, T.; REBOITA; M.S.; CARPENEDO; C.B. et al. The 2014 southeast Brazil austral summer drought: regional scale mechanisms and teleconnections. Climate Dynamics, v. 46, n. 11, p. 3737-3752, 2016.

CUADRA, S.V.; DA ROCHA, R.P. Simulação numérica do clima de verão sobre o Brasil e sua variabilidade. Revista Brasileira de Meteorologia, v. 21, n. 2, p. 271-282, 2006.

DA ROCHA, R.P. Análise Comparativa do Desempenho de Parametrizações de Convecção na Simulação da Precipitação de Verão sobre o Brasil. In: III CONGRESO CUBANO DE METEOROLOGÍA, CIUDAD DE LA HABANA, Cuba. Anais... 2005.

DA ROCHA, R.P.; REBOITA, M.S.; DUTRA, L.M.M.; LLOPART, M.P.; COPPOLA, E. Interannual variability associated with ENSO: present and future climate projections of RegCM4 for South America-CORDEX domain. Climatic Change, v. 125, p. 95-109, 2014.

DE JESUS, E.M.; DA ROCHA, R.P.; REBOITA, M.S.; LLOPART, M..; DUTRA, L.M.M.; REMEDIO, A.R.C. Contribution of cold fronts to seasonal rainfall in simulations over the southern La Plata Basin. Climate Research, v. 68, p. 243-255, 2016.

DEE D.P.; UPPALA, S.M.; SIMMONS, A.J.; BERRISFORD, P.; POLI, P. et al. The ERA-Interim reanalysis: configuration and performance of the data assimilation system. Quarterly Journal of the Royal Meteorological Society, v. 137, n. 656, p. 553-597, 2011.

DICKINSON R.E.; HENDERSON-SELLERS, A.; KENNEDY, P.J. Biosphere-atmosphere transfer scheme (BATS) version 
$1 \mathrm{E}$ as coupled to the NCAR Community Climate Model. Boulder, Colorado: Technical Note NCAR/TN-387, 72 pp, 1993.

DIRMEYER, P.A.; SCHLOSSER, C.A.; BRUBAKER, K.L. Precipitation, recycling, and land memory: an integrated analysis. Journal of Hydrometeorolology, v. 10, n. 1, p. 278288, 2009.

DOBLAS-REYES, F.J.; ANDREU-BURILLO, I.; CHIKAMOTO, Y.; GARCÍA-SERRANO, J.; GUEMAS, V. et al. Initialized near-term regional climate change prediction. Nature Comunications. V. 4, p. 1-9, 2013a.

DOBLAS-REYES, F.J.; GARCÍA-SERRANO, J.; LIENERT, F.; PINTÓ BIESCAS, A.; RODRIGUES, L.R.L. Seasonal climate predictability and forecasting: status and prospects. WIREs Climate Change, v. 4, p. 245-268, 2013 b.

ELGUINDI, N.; BI, X.; GIORGI, F.; NAGARAJAN, B.; PAL, J. et al. Regional Climate Model RegCM User Manual Version 4.4. Trieste: The Abdus Salam International Centre for Theoretical Physics. 2014.

EMANUEL, K.A.; ZIVKOVIC-ROTHMAN, M. Development and evaluation of a convection scheme for use in climate models. Journal of the Atmospheric Sciences, v. 56, p. 1766-1782, 1999.

GETTELMAN, A.; ROOD, R.B. Demystifying Climate Models: A Users Guide to Earth Systems Models. Heidelberg: Springer, v. 2, 2016.

GIORGI, F.; BATES, G.T. The climatological skill of a regional model over complex terrain. Monthly Weather Review, v. 117, p. $2325-2347,1989$.

GIORGI, F.; ANYAH, R.O. The Road Towards RegCM4. Climate Research, v. 52, p. 3-6, 2012.

GIORGI, F.; MEARNS, L.O. Introduction to special section: Regional Climate Modeling Revisited. Journal of Geophysical Research, v. 104, p. 6335-6352, 1999.

GIORGI, F.; COPPOLA, E.; SOLMON, F.; MARIOTTI, L.; SYLLA, M.B. et al. RegCM4: Model description and preliminary tests over multiple CORDEX domains. Climate Research, v. 52, p. 7-29, 2012.

GONÇALVES, J.S. Avaliação da previsão numérica sazonal de precipitação para o Rio Grande do Sul. 2015. 80 f. Dissertação (Mestrado em Meteorologia), Universidade Federal de Santa Maria, Santa Maria, 2015.

GRELL, G. A. Prognostic evaluation of assumptions used by $\mathrm{cu}$ mulus parameterization. Monthly Weather Review, v. 121, p. 764-787, 1993.

HUFFMAN, G.J.; ADLER, R.F.; BOLVIN, D.T.; GU, G.; NELKIN, E.J. et al. The TRMM Multisatellite Precipitation Analysis (TMPA): Quasi-Global, Multiyear, Combined-Sensor Precipitation Estimates at Fine Scales. Journal of Hydrometeorology, v. 8, p. 38-55, 2007.

IPCC. Summary for Policymakers. In: Climate Change 2013: The Physical Science Basis. Contribution of Working Group I to the Fifth Assessment Report of the Intergovernmental Panel on Climate Change, 2013.

KAIN, J.S. The Kain-Fritsch Convective Parameterization: An Update. American Meteorological Society. Journal of applied meteorology, v. 43, p. 170-181, 2004.

KOSTER, R.D.; SUAREZ, M.J. Soil moisture memory in climate models. Journal of Hydrometeorology, v. 2, p. 558-570, 2001.
KOSTER R.D.; MAHANAMA, S.P.P.; YAMADA T.J.; BALSAMO G.; BERG, A.A. et al. The second phase of the global land-atmosphere coupling experiment: Soil moisture contributions to subseasonal forecast skill. Journal of Hydrometeorology, v. 12, p. 805-822, 2011.

LLOPART, M.; COPPOLA, E.; GIORGI, F.; DA ROCHA, R.P.; CUADRA, S.V. Climate change impact on precipitation for the Amazon and La Plata basins. Climatic Change, v. 125, p. 111-125, 2014.

LLOPART, M.; DA ROCHA, R.P.; REBOITA, M.S.; CUADRA, S. Sensitivity of simulated South America climate to the land surface schemes in RegCM4. Climate Dynamics, v. 48, p. 1-13, 2017.

LORENZ, E.N. A study of the predictability of a 28 -variable model. Tellus, v. 17, p. 321-333, 1965.

LOVELAND, T.R.; REED, B.C.; BROWN, J.F.; OHLEN, D.O.; ZHU, Z. et al. Development of a global land cover characteristics database and IGBP DISCover from $1 \mathrm{~km}$ AVHRR data. International Journal of Remote Sensing, v. 21, n. 6-7, p. 1303-1330, 2000.

MACHADO, R.D.; DA ROCHA, R.P. Previsões climáticas sazonais sobre o Brasil: Avaliação do RegCM3 aninhado no modelo global CPTEC/COLA. Revista Brasileira de Meteorologia, v. 26, n.1, p.121-136, 2011.

MARENGO, J.A.; CAVALCANTI, I.F.A.; SATYAMURTY. P.; TROSNIKOV, I.; NOBRE, C.A. et al. Assessment of regional seasonal rainfall predictability using the CPTEC/COLA atmospheric GCM.Climate Dynamics, v. 21, p. 459-475, 2003.

MARENGO, J.A.; NOBRE, C.A.; SELUCHI, M.E.; CUARTAS, A.; ALVES, L.M.; MENDIONDO, E.M.; OBREGÓN, G.; SAMPAIO, G. A seca e a crise hídrica de 2014-2015 em São Paulo. Revista USP, n. 106, p. 31-44, 2015.

MISRA, V.; DIRMEYER, A.P.; KIRTMAN, B.P. Dynamic Downscaling of Seasonal Simulations over South America. Journal of Climate, v. 16, p. 103-117, 2003.

MOORTHI, S.; PAN, H-L.; CAPLAN, P. Changes to the 2001 NCEP operational MRF/AVN global analysis/forecast system. NWS Technical Procedures Bulletin, n. 484, pp14, 2001.

NOGUÉS-PAEGLE, J.; MO, K.C. Alternating Wet and Dry Conditions over South America during Summer. Monthly Weather Review, v. 125, p. 279-291, 1997.

ORLOWSKY, B.; SENEVIRATNE, S.I. Statistical analyses of land atmosphere feedbacks and their possible pitfalls. Journal of Climate, v. 23, p. 3918-3932, 2010.

PACANOWSKI, R.C.; GRIFFIES, S.M. MOM 3.0 Manual, NOAA/Geophysical Fluid Dynamics Laboratory, Princeton, USA 08542, 1998.

PANETTA, J.; BARROS, S.R.M.; BONATTI, J.P.; TOMITA, S.S.; KUBOTA, P.Y. Computational Coast of CPTEC AGCM. In: Proceedings of the Twelfth ECMWF Workshop - Use of High Performance Computing in Meteorology, Reading. Anais... 2006.

PRESS, W.H.; TEUKOLSKY, S.A.; VETTERLING W.T.; FLANNERY, B. P. Numerical Recipes. The art of scientific computing. Third edition, Cambridge University Press, New York, 2007.

REBOITA, M.S. Previsão Climática para a Região Sul de Minas Gerais. Relatório Científico do Projeto CNPq 473153/2010-6, 2013. 
REBOITA, M.S.; DA ROCHA, R.P.; AMBRIZZI, T. Dry and wet spells in the rainy season of the South America projected to A1B scenario by RegCM3 nested in ECHAM5. In: INTERNATIONAL CONFERENCE ON REGIONAL CLIMATE - CORDEX 2013 - WCRP-IPCC-EC joint event, Bruxelas. WCRP-IPCC-EC joint event, Anais... p. 251, 2013.

REBOITA, M.S.; DUTRA, M.M.L.; DA ROCHA, R.P.; REHBEIN, A. Dry summer over Southeastern Brazil in 2014: Part II: atmospheric patterns in ERA-Interim and simulated by CFSv2 and RegCM4 ensembles. In: 26th IUGG 2015 General Assembly, 2015, Praga. Anais 2015b.

REBOITA, M.S.; GAN, M.A.; DA ROCHA, R.P.; AMBRIZZI, T. Regimes de Precipitação na América do Sul: Uma Revisão Bibliográfica. Revista Brasileira de Meteorologia, v. 25 , n. 2 , p. $185-204,2010$.

REBOITA, M.S.; KRÜGER, L.F.; da ROCHA, R.P.; DIAS, C.G.; CUSTÓDIO, M. Validação da Precipitação Simulada pelo CFSv2. In: IV SIMPÓSIO INTERNACIONAL DE CLIMATOLOGIA, 2011, João Pessoa. Anais... 2011.

REBOITA, M.S.; FERNANDEZ, J.P.R.; LLOPART, M.P.; DA ROCHA, R.P.; PAMPUCH, L.; CRUZ, F. Assessment of RegCM4.3 over the CORDEX South America Domain: Sensitivity Analysis to the Physicals Parameterizations. Climate Research, v. 60, p. 215-234, 2014.

REBOITA, M.S.; OLIVEIRA, D.M.; FREITAS, C.H; OLIVEIRA, G.M.; PEREIRA, R.A.A. Anomalias dos Padrões Sinóticos da Atmosfera na América do Sul nos Meses de Janeiros de 2014 e 2015. Revista Brasileira de Energias Renováveis, v. 4, p. 1-12, 2015a

SAHA, S.; MOORTHI, S.; WU, X.; WANG, J.; NADIGA, S. et al. The NCEP Climate Forecast System Version 2. Journal of Climate, v. 27, n. 6, p. 2185-2208, 2014.

SENEVIRATNE, S.I; LÜTHI, D.; LITSCHI, M.; SCHÄR, C. Land-atmosphere coupling and climate change in Europe. Nature, v. 443, p. 205-209, 2006.

SILVA, G.A.M.; DUTRA, L.M.M.; DA ROCHA, R.P.; AMBRIZZI, T.; LEIVA, E. Preliminary Analysis on the Global Features of the NCEP CFSv2 Seasonal Hindcasts. Advances in Meteorology, v. 2014, p. 1-21, 2014.

VAN DEN HURK, B.; DOBLAS-REYES, F.; BALSAMO, G.; KOSTER, R.; SENEVIRATNE, S.; CAMARGO, H. Soil moisture effects on seasonal temperature and precipitation forecast scores in Europe. Climate Dynamics, v. 38, p. 349-362, 2012.

WILLMOTT, C.J. On the validation of models. Physical Geography, v. 2, p. 184-194, 1981.

XUE, Y.; SELLERS, P.J.; KINTER, J.L.; SHUKLA, J. A simplified biosphere model for global climate studies. Journal of Climate, v. 4, p. 345-364, 1991.

YNOUE, R.Y.; REBOITA, M.S.; AMBRIZZI, T.; SILVA, G.A.M. Meteorologia: Noções Básicas. Oficina de Textos, São Paulo, 2017.

\section{Sítios da internet}

Clima-CPTEC http://clima1.cptec.inpe.br/.

This is an Open Access article distributed under the terms of the Creative Commons Attribution Non-Commercial License which permits unrestricted non-commercial use, distribution, and reproduction in any medium provided the original work is properly cited. 